\title{
Asymptotic Analysis of an Elasticity Equation for a Finger-Like Hydraulic Fracture
}

\author{
José I. Adachi • Anthony P. Peirce
}

Received: 5 February 2007 / Accepted: 1 June 2007 / Published online: 17 October 2007

(C) Springer Science + Business Media B.V. 2007

\begin{abstract}
We derive a novel integral equation relating the fluid pressure in a fingerlike hydraulic fracture to the fracture width. By means of an asymptotic analysis in the small height to length ratio limit we are able to establish the action of the integral operator for receiving points that lie within three distinct regions: (1) an outer expansion region in which the dimensionless pressure is shown to be equal to the dimensionless width plus a small correction term that involves the second derivative of the width, which accounts for the nonlocal effects of the integral operator. The leading order term in this expansion is the classic local elasticity equation in the PKN model that is widely used in the oil and gas industry; (2) an inner expansion region close to the fracture tip within which the action of the elastic integral operator is shown to be the same as that of a finite Hilbert transform associated with a state of plane strain. This result will enable pressure singularities and stress intensity factors to be incorporated into analytic models of these finger-like fractures in order to model the effect of material toughness; (3) an intermediate region within which the action of the Fredholm integral operator of the first kind is reduced to a second kind operator in which the integral term appears as a small perturbation which is associated with a convergent Neumann series. These results are important for
\end{abstract}

Submitted to Journal of Elasticity on February 5, 2007. Re-submitted with revisions on May 30, 2007.

J. I. Adachi $(\varangle)$

Schlumberger Data and Consulting Services, 1325 S. Dairy Ashford Rd., Houston, TX 77077, USA

e-mail: jadachi@slb.com

A. P. Peirce

Dept. of Mathematics, The University of British Columbia,

Vancouver, BC V6T 1Z2, Canada

e-mail: peirce@math.ubc.ca 
deriving analytic models of finger-like hydraulic fractures that are consistent with linear elastic fracture mechanics.

Keywords Hydraulic fracturing • Fracture mechanics • Integral equations • Asymptotic expansions

Mathematics Subject Classifications (2000) 74B05 • 74F10 • 74R10 • 41A60 • 45E05

\section{Introduction}

Hydraulic fracturing is a process by which a fracture is propagated in a brittle material by forcing a viscous fluid into the fracture. Such a physical process occurs both naturally and by human intervention. Examples of natural hydraulic fractures occur when pressurized magma forces the formation of fractures in the earth's crust [1-3]. The deliberate generation of hydraulic fractures is typically achieved by injecting a viscous fluid into a small section of a bore-hole under a sufficiently high pressure to overcome the tensile strength of the rock as well as the far-field minimum principal geological stress. As a result, a fracture surface, which is typically assumed to be planar, develops in a direction perpendicular to the far-field minimum principal geological stress. Hydraulic fracturing is routinely used by field engineers in the oil and gas recovery industry to induce fractures in reservoirs in order to substantially enhance the flow of hydrocarbons [4-7]. Hydraulic fracturing has more recently been used in the mining industry to introduce large fractures in brittle rock to achieve more predictable and stable "caving" of the ore-body - a procedure by which large volumes of rock in the roof of a mining excavation are induced to fall into the excavation for later processing $[8,9]$. Environmental engineers have also used hydraulic fracturing to isolate toxic substances by injecting impermeable materials into fractures [10-12]. In all these processes, it is desirable to be able to predict the evolution of the fracture surface under known stress and geological conditions in order to avoid undesirable fracture penetration of environmentally sensitive regions.

Because of the sedimentary genesis of the elastic medium as well as that of the ambient geological stress field, the elastic medium is typically considered to be layered with piecewise constant elastic moduli, while the ambient geological stresses are also considered to be piecewise constant. The geometry of a hydraulic fracture as it evolves is strongly modulated by the changes of the elastic moduli between the layers and the jumps in the confining geological stresses that work to resist the formation of new fracture surface. Indeed, a typical example of the influence of the geological conditions is the formation of finger-like fractures. Such a finger-like fracture is typically caused by the containment of the evolving fracture to a single, low confinement, sedimentary layer by the existence of two high confinement layers that straddle the layer in which the fracture is propagating. These finger-like fractures are of considerable practical interest and serve as a strong motivation for the analysis presented in this paper.

The governing equations for the evolution of a fluid-driven fracture couple the Reynolds' lubrication equation, which expresses the conservation of fluid volume to the three-dimensional (3D) equilibrium and elastic stress-strain partial differential equations, that in this case can be reduced to a (2D) boundary integral equation 
expressing the balance of forces between the fluid pressure, the geological stresses, and the elastic response of the rockmass. The footprint of the fracture at any time is not known a priori, which means that the fracture's perimeter needs to be determined as part of the problem. In order to determine the location of the fracture region as the fracture evolves, an additional propagation condition is required. This condition, from linear elastic fracture mechanics, requires that the stress intensity at each point on the tip of a propagating fracture is equal to the so-called fracture toughness of the material in which that portion of the fracture tip finds itself.

The existing model for such finger-like fractures, named the PKN model after the initial researchers that developed it (see $[13,14]$ ), assumes that the fracture is much longer than it is high and that the width (i.e., crack opening displacement) is slowly varying along the length of the fracture. As a result, a state of plane strain is assumed to apply within any vertical cross-section perpendicular to the length of the fracture (see Fig. 1). Because the vertical movement of fluid within such a cross-section is minimal, the pressure within the cross-section can be assumed to be constant, which implies that the shape of the width profile is going to be elliptical. Integrating this width over the cross-section, yields a local equation which relates the pressure to the average width via a constant stiffness multiplier. This local elasticity equation has been coupled to the fluid flow equation so that the governing equation for the evolution of a finger-like hydraulic fracture is a degenerate parabolic equation of the form found in models of porous media. This approximate local elasticity equation ignores the nonlocal effects of width variations along the length of the fracture in the elasticity boundary integral equation. Because the width is zero at the tip of the fracture, the PKN fluid pressure must also approach zero at the tip. This is a serious

Fig. 1 Problem description

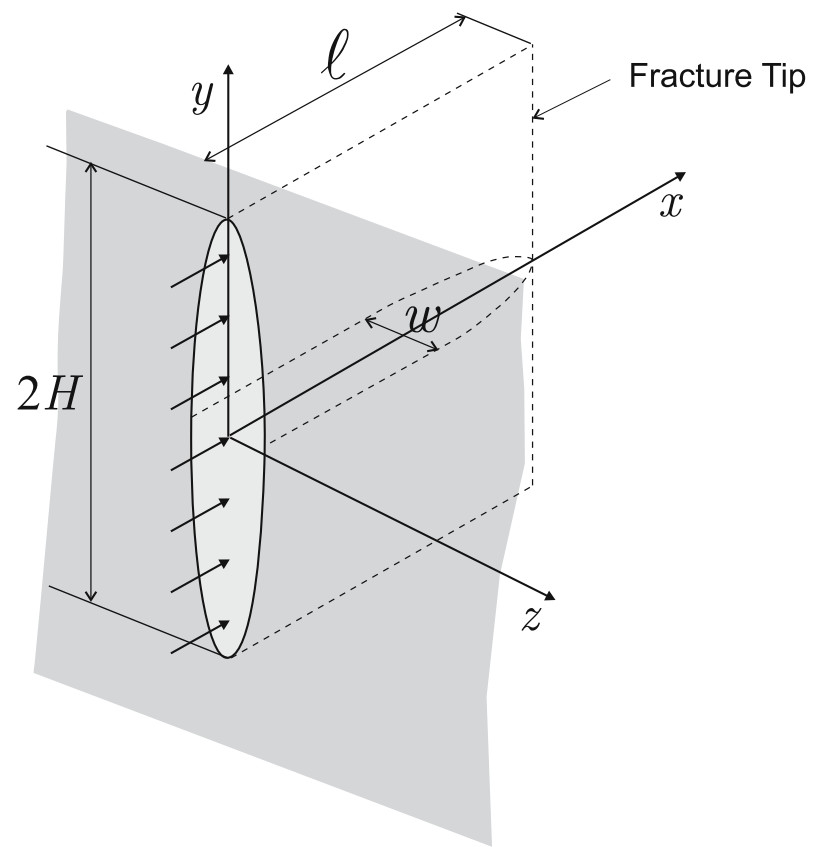


flaw in the PKN model since the regular tip pressure makes it impossible to define a stress intensity factor, which renders the model incapable of representing fracture propagation in a medium with a non-zero toughness for example.

In this paper we will describe an asymptotic analysis, in the small aspect ratio (height/length) limit, of a 2D elastic boundary integral equation for a planar fracture having a rectangular footprint. This analysis focuses only on the elasticity equations and reserves an analysis of the fully coupled hydraulic fracture equations for a later study. The objective of this paper is to reduce the $2 \mathrm{D}$ integral equation, which is only amenable to computationally intensive numerical solution, to a simplified 1D integral equation suitable to analysis as well as rapid numerical solution. Further asymptotic analysis of this 1D integral equation for points remote from the tip yields, as the leading term of an "outer expansion," the same local elasticity equation as that used in the PKN model plus a small correction term involving the second derivative of the fracture width. An asymptotic analysis of the 1D integral equation for points that are close to the tip yields an "inner expansion," which demonstrates that the action of the non-local elasticity operator is, to leading order, the same as the Finite Hilbert Transform associated with a state of plane strain. These analyses establish: (1) that the interval of applicability of the PKN approximation only includes points that are remote from the tip; (2) the appropriate correction terms to the outer expansion; and (3) the appropriate inner expansion which applies close to the tip. In order to complete the tools that are required for a matched asymptotic analysis, we require a characterization of the action of the $1 \mathrm{D}$ integral equation in an intermediate region which connects the inner solution to the outer solution. In particular, we establish that the action of the elastic Fredholm integral operator of the first kind is reduced to a second kind operator in which the integral term appears as a small perturbation. The perturbation structure of this second kind integral operator will lead to a convergent Neumann series solution.

The asymptotic analysis presented in this paper provides a rigorous justification for the PKN local equation. It also provides the fundamental formulae required for a matched asymptotic analysis of the fully coupled elasticity and lubrication equations to yield a complete asymptotic solution in which the effects of pressure singularities and fracture toughness may be included. The inclusion of this singular behavior has hitherto been missing in the analysis of finger-like fractures and is required to determine the appropriate growth conditions at the propagating fracture front.

In Section 2, we describe the classical PKN model. In Section 3, we present the $2 \mathrm{D}$ elasticity integral equation that incorporates the nonlocal effect of width variations across the length of the fracture. Assuming a constant pressure in vertical cross-sections for which the width profile is elliptic, we reduce the $2 \mathrm{D}$ hypersingular integral equation to a 1D integral equation involving a kernel that is expressed in terms of complete elliptic integrals having a Cauchy-type singularity. In Section 4, we present the asymptotic analysis of the $1 \mathrm{D}$ integral equation to establish the reduction of the action of this integral operator to local pressure-width equations or to Fredholm integral operators of the second kind in which the integral terms appear as small perturbations. In this analysis three separate expansions outer, inner, and intermediate are shown to be valid in three distinct regions relative to the fracture tips. In Section 5, we provide some concluding remarks. Detailed expressions for some of the integrals required in the asymptotic analysis are provided in appendices $\mathrm{A}, \mathrm{B}$, and $\mathrm{C}$. 


\section{The "Classical" PKN Model}

The "classical" PKN model for hydraulic fracturing [13-15] is depicted in Fig. 1: a hydraulic fracture propagates and is fully contained within a layer of constant thickness $2 H$. The model assumes that the fracture propagates only along the $x$-axis (i.e., no height-growth is allowed). In the PKN model, the following assumptions are made: (1) that the fracture length is much greater than its height; and (2) that the fracture width varies slowly along the propagation axis of the fracture. From these two assumptions, it is concluded that an approximate state of plane strain prevails in planes that are perpendicular to the propagation axis. The fluid pressure within such a vertical cross-section is approximately constant which, along with the plane strain assumption, leads to the conclusion that the shape of the fracture in any such cross-section is elliptical.

We define $w_{o}(x, t)$ to be the maximum width or opening of the fracture at any point having a coordinate $x$. The PKN model assumes that the fracture tip propagates as a "sharp front," and that the length of the fracture at any given time $t$ is given by $\ell(t)$. The net fluid pressure inside the fracture is defined as $p(x, t)=p_{\mathrm{f}}(x, t)-\sigma_{o}$, with $p_{\mathrm{f}}$ being the absolute fluid pressure and $\sigma_{o}$ the minimum in situ or confining stress acting perpendicular to the plane of the fracture. The elastic properties of the rock (which is assumed to be a homogeneous, isotropic, linear elastic material) are defined by its Young's modulus E, and Poisson's ratio v.

Assuming that the width is slowly varying across the length of the fracture, Perkins and Kern $[13,14]$ concluded that the nonlocal elasticity equation reduces to a local operator of the form

$$
p=\frac{E^{\prime}}{4 H} w_{o},
$$

where $E^{\prime}=E /\left(1-v^{2}\right)$ is the plane-strain Young's modulus.

The solution $w_{o}$ must fulfill the following conditions: (1) it must be a symmetric, continuous and smooth function; (2) it must achieve a maximum at the inlet $(x=0)$; and (3) it should be zero at the tips $(x= \pm \ell)$. Furthermore, we assume that the tip should be blunt (i.e., should not have a cusp), and that the asymptotic behavior of $w_{o}$ in the vicinity of the tip is given by

$$
w_{o} \sim\left(1 \mp \frac{x}{\ell}\right)^{\alpha}, x \rightarrow \pm \ell,
$$

where $0<\alpha<1$ is an exponent to be determined ( $\alpha$ must be less than 1 in order to ensure the tip bluntness). Due to the local nature of the elasticity equation (1), which will be made clearer in the analysis that follows, the near-tip behavior of the net fluid pressure must be identical, i.e.,

$$
p \sim\left(1 \mp \frac{x}{\ell}\right)^{\alpha}, x \rightarrow \pm \ell .
$$

\section{Formulation of the New Non-Local Elasticity Equation}

The use of the local elasticity equation (1) greatly simplifies the PKN model, hence its great popularity in the oil and gas industry. However, it is this same feature which 
makes the PKN model an "artifact" that does not fit within the rigorous theory of linear elastic fracture mechanics: the locality of the elasticity equation implies a zero pressure boundary condition at the advancing fracture tip. This boundary condition makes it impossible, for example, to define a "stress intensity factor" for the PKN model. This limitation constrains the application of the model to situations in which the toughness of the rock is negligible.

In this paper, we propose a new non-local formulation of the elasticity equation for the PKN model. To this end, we start from the general expression for the elasticity equation of a planar fracture, which is given by the hypersingular integral (e.g., [16])

$$
p(x, y)=-\lim _{z \rightarrow 0^{+}} \frac{E^{\prime}}{8 \pi} f_{S} \frac{w\left(x_{o}, y_{o}\right)}{\left[\left(x_{o}-x\right)^{2}+\left(y_{o}-y\right)^{2}+z^{2}\right]^{3 / 2}} \mathrm{~d} S,
$$

where $S$ is the footprint of the fracture in the $x-y$ plane, $E^{\prime}$ is the plane strain elastic modulus, $p$ is the net pressure, $w$ is the fracture width at any point $(x, y) \in S$, and the symbol $f$ indicates that the hypersingular integral has to be interpreted as the Hadamard finite part [17]. We will retain two assumptions of the original PKN model: (1) the shape of any vertical cross section is elliptical, i.e.,

$$
w(x, y)=w_{o}(x) \sqrt{1-\frac{y^{2}}{H^{2}}}
$$

and (2) the fluid pressure within any such cross section is constant, i.e., the fluid pressure does not depend on the $y$ coordinate. With these two assumptions, we can rewrite (3) as

$$
p(x)=-\frac{E^{\prime}}{8 \pi} f_{-\ell}^{\ell} w_{o}\left(x_{o}\right) \int_{-H}^{H} \frac{\sqrt{1-y_{o}^{2} / H^{2}}}{\left[\left(x_{o}-x\right)^{2}+y_{o}^{2}\right]^{3 / 2}} \mathrm{~d} y_{o} \mathrm{~d} x_{o} .
$$

(Notice that the inner integral is not hypersingular in $y$ any more.) Let us now introduce the following scaling

$$
\beta=\frac{H}{\ell}, \quad \xi=\frac{x}{\ell}, \quad \eta=\frac{y}{\ell}, \quad \Omega=\frac{w_{o}}{w_{*}}, \quad \Pi=p \frac{H}{E^{\prime} w_{*}},
$$

where $w_{*}$ is the proper lengthscale for the fracture width (which we will leave undefined). With this scaling, (5) reduces to

$$
\Pi(\xi)=-\frac{\beta}{8 \pi} f_{-1}^{1} \Omega\left(\xi_{o}\right) \int_{-\beta}^{\beta} \frac{\sqrt{1-\eta_{o}^{2} / \beta^{2}}}{\left[\left(\xi_{o}-\xi\right)^{2}+\eta_{o}^{2}\right]^{3 / 2}} \mathrm{~d} \eta_{o} \mathrm{~d} \xi_{o}
$$

The inner integral in (7) can be evaluated in closed-form to yield

$$
\Pi(\xi)=\frac{1}{\pi} f_{-1}^{1} \frac{\Omega\left(\xi_{o}\right)}{\left|\xi_{o}-\xi\right|}\left[\mathrm{K}\left(-\frac{\beta^{2}}{\left(\xi_{o}-\xi\right)^{2}}\right)-\mathrm{E}\left(-\frac{\beta^{2}}{\left(\xi_{o}-\xi\right)^{2}}\right)\right] \mathrm{d} \xi_{o},
$$


where $K(\cdot)$ and $E(\cdot)$ represent the complete elliptic integrals ${ }^{1}$ of the first and second kind, respectively (e.g., [18]). Integrating by parts, we can reduce (8) to the following form:

$$
\Pi(\xi)=-\frac{1}{\pi} \int_{-1}^{1} \Omega^{\prime}\left(\xi_{o}\right) G\left(\xi, \xi_{o} ; \beta\right) \mathrm{d} \xi_{o}
$$

where the kernel $G$ is defined as

$$
G\left(\xi, \xi_{o} ; \beta\right)=\operatorname{sgn}\left(\xi_{o}-\xi\right) \sqrt{1+\frac{\beta^{2}}{\left(\xi_{o}-\xi\right)^{2}}} \mathrm{E}\left(\frac{\beta^{2}}{\left(\xi_{o}-\xi\right)^{2}+\beta^{2}}\right),
$$

with $\operatorname{sgn}(\cdot)$ representing the sign of the argument and ()$^{\prime}$ indicating derivative with respect to the argument. Notice that the integral above is not hypersingular anymore, but just singular in the Cauchy Principal Value sense, whence the symbol $f$ in (9).

In deriving the above, we have assumed that $\Omega(\xi= \pm 1)=0$ and that $\Omega$ (the dimensionless fracture opening) is a positive, symmetric (even) function which is sufficiently smooth in $\xi \in(-1,1)$ that the integral in $(9)$ exists. We also introduce the additional assumption that the fracture tips are blunt, i.e., that the asymptotic behavior of the function $\Omega$ close to the tips is of the form $\Omega \sim(1 \mp \xi)^{\alpha}$, $\xi \rightarrow \pm 1$, with $0<\alpha<1$.

\section{Asymptotic Analysis of the New Elasticity Equation}

\subsection{Asymptotic Expansions of the Elasticity Kernel}

In this section, we perform an asymptotic analysis of the elasticity equation (9) assuming the dimensionless ratio $\beta=H / \ell \ll 1$. The reason for selecting $\beta$ as our small parameter comes naturally from the fact that we are considering the case of a PKN-type fracture, which is characterized by such a small aspect ratio.

If we define $\rho=\left|\xi_{o}-\xi\right| / \beta$, the kernel $G$ can be expressed as

$$
G(\rho)=\frac{1}{\rho} \sqrt{\rho^{2}+1} \mathrm{E}\left(\frac{1}{\rho^{2}+1}\right) .
$$

Performing asymptotic expansions for small ${ }^{2}$ and large values of $\rho$, we obtain

$$
G(\rho) \sim \frac{1}{\rho}-\frac{1}{2} \rho \ln \rho+\left(\frac{1}{4}+\ln 2\right) \rho+O\left(\rho^{3} \ln \rho\right), \rho \ll 1,
$$

and

$$
G(\rho) \sim \frac{\pi}{2}\left[1+\frac{1}{4} \rho^{-2}-\frac{3}{64} \rho^{-4}\right]+O\left(\rho^{-6}\right), \rho \gg 1 .
$$

\footnotetext{
${ }^{1}$ Notice that for the arguments of the complete elliptic integrals we are using the convention used in [18], i.e., $\mathrm{K}(m)=\int_{0}^{\pi / 2}\left(1-m \sin ^{2} \theta\right)^{-1 / 2} \mathrm{~d} \theta$ and $\mathrm{E}(m)=\int_{0}^{\pi / 2}\left(1-m \sin ^{2} \theta\right)^{1 / 2} \mathrm{~d} \theta$.

${ }^{2}$ Expansion (12) can be obtained from the identity $\mathrm{E}(z)=\frac{1}{2}\left[Q_{-1 / 2}(2 z-1)-Q_{1 / 2}(2 z-1)\right]$, where $Q_{v}(\cdot)$ is the Legendre function of the second kind, of degree $v[19]$.
} 
Fig. 2 Plot of the kernel $G(\rho)$ (solid line) versus

$\rho=\left|\xi_{o}-\xi\right| / \beta$. The asymptotic expansions (12) (dotted line to the left) and (13) (dashed line to the right) are plotted for comparison. The first terms of both expansions are also plotted

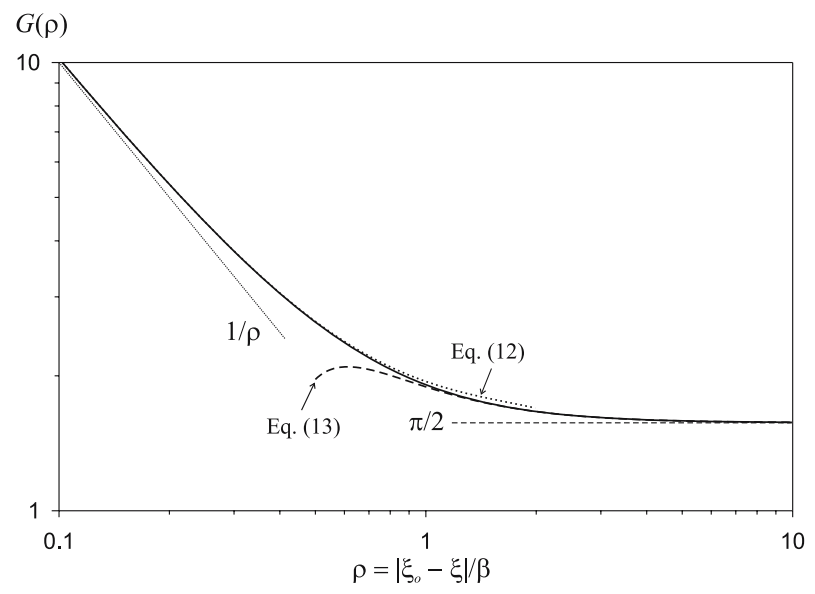

These expansions, when truncated after the third term, are actually valid up to $\rho \sim 1$. Figure 2 shows a comparison between the exact kernel and the two expansions. Figure 3 shows the relative error between the expansions and the exact kernel. For both truncated expansions, the relative error at $\rho=1$ is about $1 \%$. (In these plots, the complete elliptic integral of the second kind was computed using the software Mathematica $^{\mathrm{TM}}$, version 4.1 [ [C 1988-2000 Wolfram Research].)

The above results allow us to reach very interesting conclusions. From (12) and Fig. 2, it is quite clear that within a region $\xi_{o} \in[\xi-\beta / 10, \xi+\beta / 10]$, the behavior of the elastic kernel is practically dominated by the term $1 / \rho=\beta /\left|\xi_{o}-\xi\right|$, which we know to be the classical plane-strain singularity of the elasticity kernel. For $\left|\xi_{o}-\xi\right| \gtrsim 10 \beta$, on the other hand, we have that the kernel starts to be dominated by a constant term, i.e., we start to recover the "classical" PKN behavior, in the sense of a local dependency between pressure and width.

Due to expected singularities of the fracture opening gradient $\Omega^{\prime}$ at the fracture tips (recall that we are assuming that $\Omega \sim(1 \mp \xi)^{\alpha}$ as $\xi \rightarrow \pm 1$, with $0<\alpha<1$ ), special care has to be given to the analysis of (9) in the vicinity of the tips. For this reason, we have sub-divided the present analysis into three parts, that we call "outer," "intermediate" and "inner."

Fig. 3 Plot of relative error for the asymptotic expansions (12) (dotted line) and (13) (dashed line), versus $\rho=\left|\xi_{o}-\xi\right| / \beta$. Both expansions are truncated at the third term

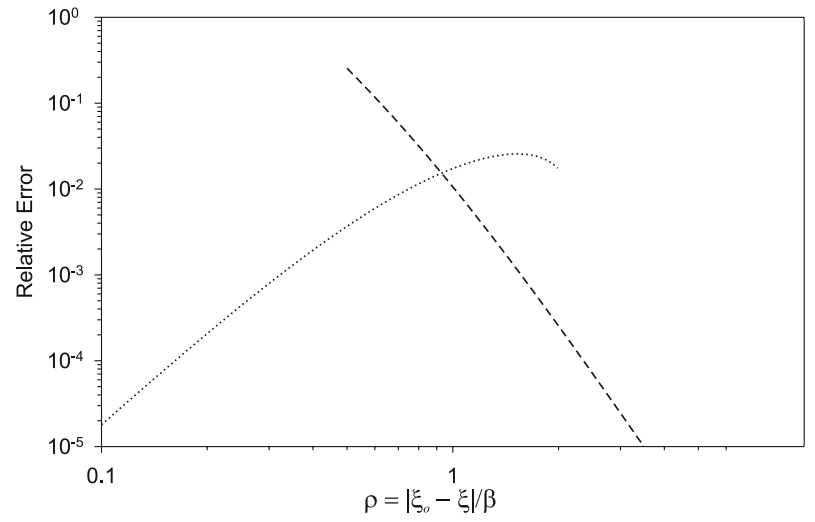




\subsection{Outer Expansion}

Let us first consider the case of a point $\xi$ located "away from the tips," which we define as $|\xi|<1-\beta$. For this case, we divide the problem domain $[-1,1]$ into the following sectors:

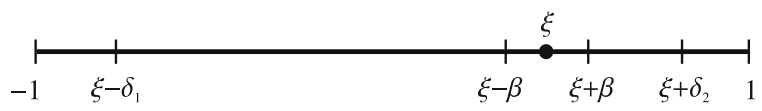

where $\delta_{1}$ and $\delta_{2}$ are positive numbers, defined such that the following asymptotic behavior for $\Omega$ is valid

$$
\begin{array}{ll}
\Omega\left(\xi_{o}\right) \sim A\left(1+\xi_{o}\right)^{\alpha}, & \xi_{o} \in\left[-1, \xi-\delta_{1}\right], \\
\Omega\left(\xi_{o}\right) \sim A\left(1-\xi_{o}\right)^{\alpha}, & \xi_{o} \in\left[\xi+\delta_{2}, 1\right] .
\end{array}
$$

In the region defined by $\xi_{o} \in\left[\xi-\delta_{1}, \xi+\delta_{2}\right]$, on the other hand, we assume that the gradient $\Omega^{\prime}$ can be approximated using a Taylor series expansion, i.e.,

$\Omega^{\prime}\left(\xi_{o}\right) \sim \Omega^{\prime}(\xi)+\left(\xi_{o}-\xi\right) \Omega^{\prime \prime}(\xi)+\frac{1}{2}\left(\xi_{o}-\xi\right)^{2} \Omega^{\prime \prime \prime}(\xi)+\ldots, \quad \xi_{o} \in\left[\xi-\delta_{1}, \xi+\delta_{2}\right]$.

The expansions (12) and (13) indicate that we can divide the integral in (9) into three regions: a Cauchy Principal Value region $\xi_{o} \in[\xi-\beta, \xi+\beta]$, within which we can approximate the kernel using the expansion (12), and two other regions defined by $\xi_{o} \in[-1, \xi-\beta]$ and $\xi_{o} \in[\xi+\beta, 1]$, within which the kernel can be approximated using the expansion (13). Proceeding in this way (9) can be expressed in the form:

$$
\begin{aligned}
\Pi(\xi ; \beta)= & \frac{1}{2}[\Omega(\xi-\beta)+\Omega(\xi+\beta)]+\int_{\xi-\beta}^{\xi+\beta} \Omega^{\prime}\left(\xi_{o}\right) G_{1}\left(\xi, \xi_{o}\right) \mathrm{d} \xi_{o} \\
& +\beta^{2}\left[\int_{-1}^{\xi-\beta} \Omega^{\prime}\left(\xi_{o}\right) G_{2}\left(\xi, \xi_{o}\right) \mathrm{d} \xi_{o}-\int_{\xi+\beta}^{1} \Omega^{\prime}\left(\xi_{o}\right) G_{2}\left(\xi, \xi_{o}\right) \mathrm{d} \xi_{o}\right],
\end{aligned}
$$

with the kernels $G_{1}$ and $G_{2}$ defined as

$$
\begin{aligned}
& G_{1}\left(\xi, \xi_{o}\right)=-\frac{1}{\pi}\left[\frac{\beta}{\xi_{o}-\xi}-\frac{1}{2 \beta}\left(\xi_{o}-\xi\right) \ln \left|\frac{\xi_{o}-\xi}{\beta}\right|+\frac{1}{\beta}\left(\frac{1}{4}+\ln 2\right)\left(\xi_{o}-\xi\right)\right], \\
& G_{2}\left(\xi, \xi_{o}\right)=\frac{1}{8}\left[\frac{1}{\left(\xi_{o}-\xi\right)^{2}}-\frac{3 \beta^{2}}{16\left(\xi_{o}-\xi\right)^{4}}\right] .
\end{aligned}
$$

We first notice that if $\beta \ll 1$ and $[\xi-\beta, \xi+\beta] \subset\left[\xi-\delta_{1}, \xi+\delta_{2}\right]$, it is valid to approximate the first term in (16) as follows:

$$
\frac{1}{2}[\Omega(\xi-\beta)+\Omega(\xi+\beta)] \simeq \Omega(\xi)+\frac{1}{2} \beta^{2} \Omega^{\prime \prime}(\xi) .
$$

Let us define the integrals $I_{1}^{\mathrm{OUT}}$ and $I_{2}^{\mathrm{OUT}}$ as

$$
\begin{aligned}
& I_{1}^{\mathrm{OUT}}(\xi ; \beta)=\int_{\xi-\beta}^{\xi+\beta} \Omega^{\prime}\left(\xi_{o}\right) G_{1}\left(\xi, \xi_{o}\right) \mathrm{d} \xi_{o} \\
& I_{2}^{\mathrm{OUT}}(\xi ; \beta)=\beta^{2}\left[\int_{-1}^{\xi-\beta} \Omega^{\prime}\left(\xi_{o}\right) G_{2}\left(\xi, \xi_{o}\right) \mathrm{d} \xi_{o}-\int_{\xi+\beta}^{1} \Omega^{\prime}\left(\xi_{o}\right) G_{2}\left(\xi, \xi_{o}\right) \mathrm{d} \xi_{o}\right] .
\end{aligned}
$$


Provided $\beta \ll 1$ and assuming that the interval $[\xi-\beta, \xi+\beta]$ is completely contained within the interval $\left[\xi-\delta_{1}, \xi+\delta_{2}\right]$, integration of $I_{1}^{\text {OUT }}$ yields (for details, see Appendix A)

$$
I_{1}^{\text {OUT }}(\xi ; \beta)=-\frac{\beta^{2}}{\pi}\left(\frac{41}{18}+\frac{2}{3} \ln 2\right) \Omega^{\prime \prime}(\xi)+O\left(\beta^{4}\right), \quad|\xi|<1-\beta .
$$

This is the correction coming from inside the Cauchy principal value region, dominated by the asymptote (12). For $I_{2}^{\mathrm{OUT}}$, on the other hand, let us define

$$
I_{2}^{\mathrm{OUT}}(\xi ; \beta)=\frac{1}{8} \beta^{2} I_{21}^{\mathrm{OUT}}(\xi ; \beta)-\frac{3}{128} \beta^{4} I_{22}^{\mathrm{OUT}}(\xi ; \beta),
$$

with $I_{21}^{\mathrm{OUT}}$ and $I_{22}^{\mathrm{OUT}}$ given by

$$
\begin{aligned}
I_{21}^{\mathrm{OUT}}(\xi ; \beta)= & A \alpha \int_{-1}^{\xi-\delta_{1}} \frac{\left(1+\xi_{o}\right)^{\alpha-1}}{\left(\xi_{o}-\xi\right)^{2}} \mathrm{~d} \xi_{o}+\int_{\xi-\delta_{1}}^{\xi-\beta} \frac{\Omega^{\prime}\left(\xi_{o}\right)}{\left(\xi_{o}-\xi\right)^{2}} \mathrm{~d} \xi_{o} \\
& -\int_{\xi+\beta}^{\xi+\delta_{2}} \frac{\Omega^{\prime}\left(\xi_{o}\right)}{\left(\xi_{o}-\xi\right)^{2}} \mathrm{~d} \xi_{o}+A \alpha \int_{\xi+\delta_{2}}^{1} \frac{\left(1-\xi_{o}\right)^{\alpha-1}}{\left(\xi_{o}-\xi\right)^{2}} \mathrm{~d} \xi_{o}, \\
I_{22}^{\mathrm{OUT}}(\xi ; \beta)= & A \alpha \int_{-1}^{\xi-\delta_{1}} \frac{\left(1+\xi_{o}\right)^{\alpha-1}}{\left(\xi_{o}-\xi\right)^{4}} \mathrm{~d} \xi_{o}+\int_{\xi-\delta_{1}}^{\xi-\beta} \frac{\Omega^{\prime}\left(\xi_{o}\right)}{\left(\xi_{o}-\xi\right)^{4}} \mathrm{~d} \xi_{o} \\
& -\int_{\xi+\beta}^{\xi+\delta_{2}} \frac{\Omega^{\prime}\left(\xi_{o}\right)}{\left(\xi_{o}-\xi\right)^{4}} \mathrm{~d} \xi_{o}+A \alpha \int_{\xi+\delta_{2}}^{1} \frac{\left(1-\xi_{o}\right)^{\alpha-1}}{\left(\xi_{o}-\xi\right)^{4}} \mathrm{~d} \xi_{o} .
\end{aligned}
$$

Let us first focus on $I_{21}^{\mathrm{OUT}}$. The first and last integrals in (22a) can be evaluated in closed form to yield:

$$
\begin{aligned}
\int_{-1}^{\xi-\delta_{1}} \frac{\left(1+\xi_{o}\right)^{\alpha-1}}{\left(\xi_{o}-\xi\right)^{2}} \mathrm{~d} \xi_{o} & =\frac{\left(1+\xi-\delta_{1}\right)^{\alpha}}{\delta_{1}(1+\xi)}\left[1+\left(\frac{1-\alpha}{\alpha}\right){ }_{2} \mathrm{~F}_{1}\left(1,1 ; \alpha+1 ; 1-\frac{1+\xi}{\delta_{1}}\right)\right], \\
\int_{\xi+\delta_{2}}^{1} \frac{\left(1-\xi_{o}\right)^{\alpha-1}}{\left(\xi_{o}-\xi\right)^{2}} \mathrm{~d} \xi_{o} & =\frac{\left(1-\xi-\delta_{2}\right)^{\alpha}}{\delta_{2}(1-\xi)}\left[1+\left(\frac{1-\alpha}{\alpha}\right){ }_{2} \mathrm{~F}_{1}\left(1,1 ; \alpha+1 ; 1-\frac{1-\xi}{\delta_{2}}\right)\right],
\end{aligned}
$$

where ${ }_{2} \mathrm{~F}_{1}(\cdot, \cdot ; \cdot ; \cdot)$ is Gauss' hypergeometric function [18]. Let us now define the numbers $\epsilon_{1}$ and $\epsilon_{2}$ in the following way

$$
\epsilon_{1}=\frac{\delta_{1}}{1+\xi}, \quad \epsilon_{2}=\frac{\delta_{2}}{1-\xi},
$$

such that $\epsilon_{1}<1$ and $\epsilon_{2}<1$. Expanding the results in (23) in terms of small $\epsilon_{1}$ and $\epsilon_{2}$, we obtain

$$
\begin{gathered}
\int_{-1}^{\xi-\delta_{1}} \frac{\left(1+\xi_{o}\right)^{\alpha-1}}{\left(\xi_{o}-\xi\right)^{2}} \mathrm{~d} \xi_{o} \sim \frac{1}{\epsilon_{1}}(1+\xi)^{\alpha-2}+\phi(\alpha)(1+\xi)^{\alpha-2}+O\left(\epsilon_{1}\right), \\
\int_{\xi+\delta_{2}}^{1} \frac{\left(1-\xi_{o}\right)^{\alpha-1}}{\left(\xi_{o}-\xi\right)^{2}} \mathrm{~d} \xi_{o} \sim \frac{1}{\epsilon_{2}}(1-\xi)^{\alpha-2}+\phi(\alpha)(1-\xi)^{\alpha-2}+O\left(\epsilon_{2}\right),
\end{gathered}
$$


where the coefficient $\phi$ is defined as

$$
\phi(\alpha)=(\alpha-1)(\gamma+\psi(\alpha))-\alpha,
$$

with $\psi(\cdot)$ being the digamma function [18], and $\gamma \simeq 0.577216$ is Euler's constant. Notice that we can re-write the above results in the following form

$$
\begin{gathered}
\int_{-1}^{\xi-\delta_{1}} \frac{\left(1+\xi_{o}\right)^{\alpha-1}}{\left(\xi_{o}-\xi\right)^{2}} \mathrm{~d} \xi_{o} \sim \phi(\alpha)(1+\xi)^{\alpha-2}+\frac{1}{\alpha A} g_{11}\left(\xi ; \alpha, \delta_{1}\right)+O\left(\epsilon_{1}\right), \\
\int_{\xi+\delta_{2}}^{1} \frac{\left(1-\xi_{o}\right)^{\alpha-1}}{\left(\xi_{o}-\xi\right)^{2}} \mathrm{~d} \xi_{o} \sim \phi(\alpha)(1-\xi)^{\alpha-2}+\frac{1}{\alpha A} g_{12}\left(\xi ; \alpha, \delta_{2}\right)+O\left(\epsilon_{2}\right),
\end{gathered}
$$

with $g_{11}$ and $g_{12}$ being functions that depend upon $\delta_{1}$ and $\delta_{2}$, respectively.

The second and third integrals in (22a) can be approximated using the Taylor series expansion for $\Omega^{\prime}$. For the first integral, we find

$$
\int_{\xi-\delta_{1}}^{\xi-\beta} \frac{\Omega^{\prime}\left(\xi_{o}\right)}{\left(\xi_{o}-\xi\right)^{2}} \mathrm{~d} \xi_{o} \sim \Omega^{\prime}(\xi) \int_{\xi-\delta_{1}}^{\xi-\beta} \frac{\mathrm{d} \xi_{o}}{\left(\xi_{o}-\xi\right)^{2}}+\Omega^{\prime \prime}(\xi) \int_{\xi-\delta_{1}}^{\xi-\beta} \frac{\mathrm{d} \xi_{o}}{\xi_{o}-\xi}+\ldots
$$

which yields

$$
\int_{\xi-\delta_{1}}^{\xi-\beta} \frac{\Omega^{\prime}\left(\xi_{o}\right)}{\left(\xi_{o}-\xi\right)^{2}} d \xi_{o} \sim \frac{1}{\beta} \Omega^{\prime}(\xi)+\ln \beta \Omega^{\prime \prime}(\xi)-\frac{1}{2} \beta \Omega^{\prime \prime \prime}(\xi)-g_{11}\left(\xi ; \alpha, \delta_{1}\right)+O(\beta \ln \beta) .
$$

The function $g_{11}$ above is the same one that appears in the expansion (26a). Recall that $\delta_{1}$ and $\delta_{2}$ are arbitrary parameters, whose purpose is to identify the regions within which both the Taylor series expansion, and the near-tip asymptotic behaviors of $\Omega^{\prime}$ overlap. In other words, these numbers are introduced just to mark the boundaries between two distinct expansion regions. Thus both $\delta_{1}$ and $\delta_{2}$ can be considered as artificial parameters, and the final result of the integral should not depend on either value. Hence, for the asymptotic expansion to be valid, we require that $g_{11}$ and $g_{12}$ cancel out when adding all the components of the integral. This can be verified by direct computation.

The same procedure can be repeated for the third integral, in which case we find

$$
\int_{\xi+\beta}^{\xi+\delta_{2}} \frac{\Omega^{\prime}\left(\xi_{o}\right)}{\left(\xi_{o}-\xi\right)^{2}} \mathrm{~d} \xi_{o} \sim \frac{1}{\beta} \Omega^{\prime}(\xi)-\ln \beta \Omega^{\prime \prime}(\xi)-\frac{1}{2} \beta \Omega^{\prime \prime \prime}(\xi)+g_{12}\left(\xi ; \alpha, \delta_{2}\right)+O(\beta \ln \beta),
$$

where again, $g_{12}$ is the same function that appears in (26b). Combining (26), (27) and (28) according to (22a), we finally obtain

$$
I_{21}^{\mathrm{OUT}}(\xi ; \beta) \sim 2 \ln \beta \Omega^{\prime \prime}(\xi)+\alpha A \phi(\alpha)\left[(1+\xi)^{\alpha-2}+(1-\xi)^{\alpha-2}\right]+O(\beta)
$$


A similar procedure can be used to determine the asymptotic expansion in $\beta$ for $I_{22}^{\text {OUT }}$ [as defined in (22b)]. In general, we can express

$$
\begin{gathered}
\int_{-1}^{\xi-\delta_{1}} \frac{\left(1+\xi_{o}\right)^{\alpha-1}}{\left(\xi_{o}-\xi\right)^{4}} \mathrm{~d} \xi_{o} \sim f_{21}(\xi ; \alpha)+\frac{1}{\alpha A} g_{21}\left(\xi ; \alpha, \delta_{1}\right)+O\left(\epsilon_{1}\right), \\
\int_{\xi+\delta_{2}}^{1} \frac{\left(1-\xi_{o}\right)^{\alpha-1}}{\left(\xi_{o}-\xi\right)^{4}} \mathrm{~d} \xi_{o} \sim f_{22}(\xi ; \alpha)+\frac{1}{\alpha A} g_{22}\left(\xi ; \alpha, \delta_{2}\right)+O\left(\epsilon_{2}\right) .
\end{gathered}
$$

(We leave $f_{21}$ and $f_{22}$ undefined for reasons that will become clear later.) Also, we obtain

$$
\begin{aligned}
& \int_{\xi-\delta_{1}}^{\xi-\beta} \frac{\Omega^{\prime}\left(\xi_{o}\right)}{\left(\xi_{o}-\xi\right)^{4}} \mathrm{~d} \xi_{o} \sim \frac{1}{3 \beta^{3}} \Omega^{\prime}(\xi)-\frac{1}{2 \beta^{2}} \Omega^{\prime \prime}(\xi)+\frac{1}{2 \beta} \Omega^{\prime \prime \prime}(\xi)-g_{21}\left(\xi ; \alpha, \delta_{1}\right)+O(\ln \beta), \\
& \int_{\xi+\beta}^{\xi+\delta_{2}} \frac{\Omega^{\prime}\left(\xi_{o}\right)}{\left(\xi_{o}-\xi\right)^{4}} \mathrm{~d} \xi_{o} \sim \frac{1}{3 \beta^{3}} \Omega^{\prime}(\xi)+\frac{1}{2 \beta^{2}} \Omega^{\prime \prime}(\xi)+\frac{1}{2 \beta} \Omega^{\prime \prime \prime}(\xi)+g_{22}\left(\xi ; \alpha, \delta_{2}\right)+O(\ln \beta),
\end{aligned}
$$

from which we obtain

$$
I_{22}^{\mathrm{OUT}}(\xi ; \beta) \sim-\frac{1}{\beta^{2}} \Omega^{\prime \prime}(\xi)+\alpha A\left[f_{21}(\xi ; \alpha)+f_{22}(\xi ; \alpha)\right]+O(\ln \beta) .
$$

Combination of (29) and (30) according to (21) yields, after truncating terms of order higher than $\beta^{2}$,

$$
\begin{aligned}
I_{2}^{\mathrm{OUT}}(\xi ; \beta)= & -\frac{1}{4} \beta^{2} \ln \beta \Omega^{\prime \prime}(\xi)+\frac{67}{128} \beta^{2} \Omega^{\prime \prime}(\xi) \\
& +\frac{1}{8} \beta^{2} \alpha A \phi(\alpha)\left[(1+\xi)^{\alpha-2}+(1-\xi)^{\alpha-2}\right] .
\end{aligned}
$$

Notice that the term $\alpha A\left[f_{21}(\xi ; \alpha)+f_{22}(\xi ; \alpha)\right]$ is not included in the above expansion, as this term is of $O\left(\beta^{4}\right)$. Finally, by combining (18), (20) and (31) according to (16), we obtain the outer expansion of the elastic integral equation (9), which is valid in the regions remote from the tip:

$$
\begin{aligned}
\Pi^{\mathrm{OUT}}(\xi ; \beta)= & \Omega(\xi)+\frac{1}{4} \beta^{2} \ln \beta \Omega^{\prime \prime}(\xi)+\beta^{2}\left[\frac{67}{128}-\frac{1}{\pi}\left(\frac{41}{18}+\frac{2}{3} \ln 2\right)\right] \Omega^{\prime \prime}(\xi) \\
& +\frac{1}{8} \beta^{2} \alpha A \phi(\alpha)\left[(1+\xi)^{\alpha-2}+(1-\xi)^{\alpha-2}\right]+O(\beta), \quad|\xi|<1-\beta .
\end{aligned}
$$

We observe that equating $\Pi^{\text {OUT }}$ to the first order term in (32) yields the dimensionless form of the PKN local elasticity equation (1). The subsequent terms in the expansion represent the correction to the PKN elasticity equation in which the higher derivatives of $\Omega$ account for the nonlocal effects due to the variations in the width. When combined with the fluid flow equation this correction, involving the second order derivatives of $\Omega$, will change the hydraulic fracture evolution equation from a porous medium equation to a fourth order partial differential equation multiplied by the small parameter $\beta^{2} \ln \beta$. Investigating the properties of this equation are beyond the scope of this paper.

A plot comparing the asymptotic expansion in (32) with a numerical evaluation of the elasticity integral equation (9) is shown in Fig. 4. To generate this plot, we have 
Fig. 4 Plot of the dimensionless pressure $\Pi$ calculated numerically from (9) $($ dots $)$ versus normalized distance from the tip $(1-\xi) / \beta$. Comparison between $\Pi$, the outer expansion (32) (solid line), and the dimensionless fracture opening $\Omega$ (dashed line). Results were obtained using the test function $\Omega=\left(1-\xi^{2}\right)^{2 / 3}$ and $\beta=0.01$

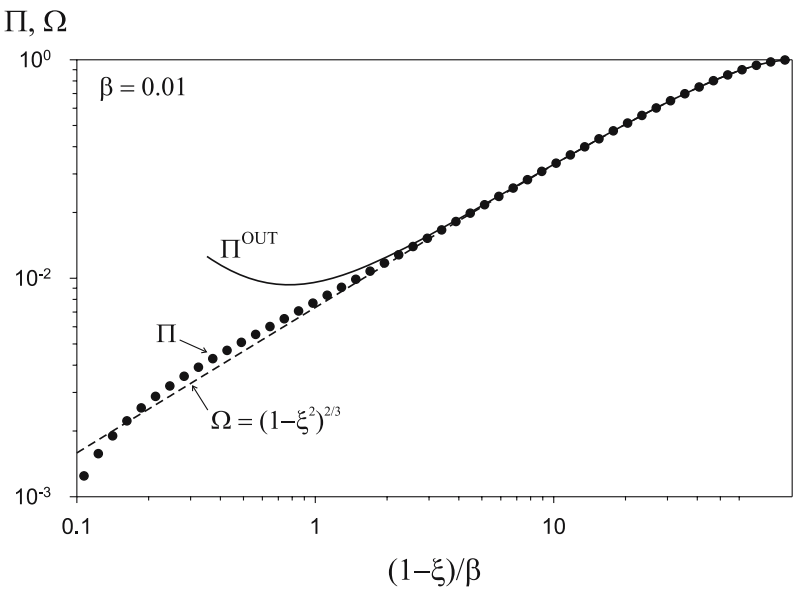

used a "test function" for $\Omega$, of the form $\Omega=\left(1-\xi^{2}\right)^{2 / 3}$ (i.e., $\alpha=2 / 3$ and $A=2^{2 / 3}$ ). The calculations were performed using $\beta=0.01$. In this plot, we observe that the outer expansion has a range of validity of $3 \beta$ to $100 \beta$ from the tip (in the plotted example, $100 \beta$ corresponds to the fracture inlet, i.e., the center of the fracture). The divergence of the outer asymptotic expansion as we approach the fracture tip can be explained by the fact that the Taylor expansion of $\Omega^{\prime}(\xi)$ only has a radius of convergence of $|1-\xi|$ due to the singularity of $\Omega^{\prime}(\xi)$ at the tip $\xi=1$. Thus as $\xi$ approaches the tip, the slow convergence of the Taylor expansion means that many more terms are required to accurately estimate the integrals $I_{21}^{\mathrm{OUT}}$ and $I_{22}^{\mathrm{OUT}}$. This is the cause of the loss of accuracy of the three-term expansion $\Pi^{\text {OUT }}$ in the region $\xi<1-3 \beta$. From Fig. 4 we can see that the leading order term in the outer solution $\Pi \approx \Omega$ (which is just the classic PKN solution) is valid over the larger interval $1-\beta<\xi<100 \beta$ than the three-term outer expansion (32). Based on the plots shown in Fig. 4 it may seem preferable to merely use the leading order term in the outer expansion. However, if we compare the errors in the one-term and the threeterm outer expansions as shown in Fig. 5, we observe that the three term expansion

Fig. 5 Plot of the relative difference between dimensionless net pressure $\Pi$ and the outer expansion (32) versus normalized distance from the tip $(1-\xi) / \beta$ (solid line), compared to the difference between $\Pi$ and dimensionless fracture opening $\Omega$ (dashed line). Results were obtained using the test function $\Omega=\left(1-\xi^{2}\right)^{2 / 3}$ and $\beta=0.01$

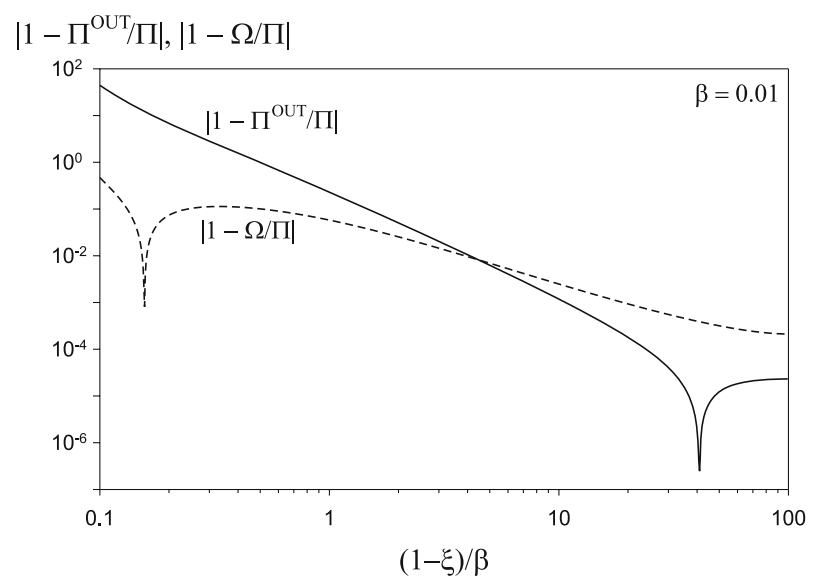


is indeed more accurate in the region $1-3 \beta<\xi<100 \beta$. Thus for leading order asymptotics, the solution $\Pi=\Omega$ can be used over the interval $1-\beta<\xi<100 \beta$ and matched to an inner solution at the point $\xi=1-\beta$. However, for higher order asymptotics, it will be necessary to introduce an intermediate region $1-\beta<\xi<3 \beta$ within which an alternative description of the action of the integral operator is required. The solution in the intermediate region can then be matched to the higher order inner and outer solutions at the points $1-\beta$ and $1-3 \beta$, respectively.

\subsection{Inner Expansion}

The partition of the integration domain used for the outer expansion is only valid for $|\xi|<1-\beta$. It is clear that if $\xi$ is closer than a distance $\beta$ from either of the fracture tips, then the principal values in (19a) as derived in Appendix A are no longer valid, for two reasons: (1) the integration interval losses symmetry with respect to $\xi$; and (2) the approximation of $\Omega^{\prime}$ by a Taylor series expansion may not be applicable because of the bluntness assumption for $\Omega$. In this case, considering for time being only the right tip $\xi=1$, we propose to sub-divide the integration domain in the following way:

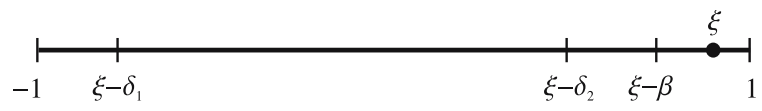

Similar to the case of the outer expansion, we postulate that $\delta_{1}$ and $\delta_{2}$ mark the boundaries in which the asymptotic behaviors described by (14) and (15) are valid. The region in which the kernel expansion (12) is valid (the Cauchy Principal Value region) is now given by $\xi_{o} \in[\xi-\beta, 1]$. We can thus define the integral $I_{1}^{\text {in }}$ as

$$
I_{1}^{\text {in }}(\xi ; \beta)=f_{\xi-\beta}^{1} \Omega^{\prime}\left(\xi_{o}\right) G_{1}\left(\xi, \xi_{o}\right) \mathrm{d} \xi_{o}, \xi>1-\beta,
$$

and the integral $I_{2}^{\text {in }}$ as

$$
I_{2}^{\text {in }}(\xi ; \beta)=\frac{1}{2} \Omega(\xi-\beta)+\beta^{2} \int_{-1}^{\xi-\beta} \Omega^{\prime}\left(\xi_{o}\right) G_{2}\left(\xi, \xi_{o}\right) \mathrm{d} \xi_{o}, \xi>1-\beta,
$$

with the kernels $G_{1}$ and $G_{2}$ as defined in (17). To evaluate $I_{1}^{\text {in }}$, it is easier to first re-scale the problem in terms of a stretched system of coordinates $\hat{\xi}$ with origin at either of the fracture tips, and pointing inwards to the fracture center. Taking the tip $\xi=1$, we define in the usual way

$$
\hat{\xi}=\beta^{-1}(1-\xi) .
$$

The region $\xi>1-\beta$ is now defined by $\hat{\xi}<1$. We are also assuming that $\beta$ is small enough that $[\xi-\beta, 1] \subset\left[\xi-\delta_{2}, 1\right]$. Hence, in the new scaling, and considering (14), we have $\hat{\Omega} \sim A \hat{\xi}^{\alpha}, \hat{\xi} \rightarrow 0$. Following this, let us propose the following re-scaling for both $\Omega$ and $\Pi$

$$
\hat{\Omega}(\hat{\xi})=\beta^{-\alpha} \Omega(\xi), \quad \hat{\Pi}=\beta^{-\alpha} \Pi(\xi),
$$


where $\alpha$ is, in this case, the same exponent that defines the asymptotic behavior of $\Omega$ near the tips. In this new scaling, (33) can be re-written as follows

$$
\begin{aligned}
J_{1}(\hat{\xi}) & =\frac{\alpha A}{\pi} f_{0}^{\hat{\xi}+1} \hat{\xi}_{o}^{\alpha-1}\left[\frac{1}{\hat{\xi}_{o}-\hat{\xi}}-\frac{1}{2}\left(\hat{\xi}_{o}-\hat{\xi}\right) \ln \left|\hat{\xi}_{o}-\hat{\xi}\right|+\left(\frac{1}{4}+\ln 2\right)\left(\hat{\xi}_{o}-\hat{\xi}\right)\right] \mathrm{d} \hat{\xi}_{o}, \\
\hat{\xi} & <1
\end{aligned}
$$

where $J_{1}=\beta^{-\alpha} I_{1}^{\text {in }}$. All the integrals in $J_{1}$ can be calculated in closed-form, and the results are listed in Appendix B. An expansion of $J_{1}(\hat{\xi})$ for $\hat{\xi} \ll 1$ (i.e., for $1-\xi \ll \beta$ ) yields

$$
\frac{\pi}{\alpha A} J_{1}(\hat{\xi}) \sim \pi \cot \pi \alpha \hat{\xi}^{\alpha-1}+\lambda_{0}+\lambda_{1} \hat{\xi}+O\left(\hat{\xi}^{\alpha+1}\right)
$$

where the coefficients $\lambda_{0}$ and $\lambda_{1}$ are given by

$$
\begin{aligned}
& \lambda_{0}=\frac{1}{1-\alpha}-\frac{1}{2(1+\alpha)^{2}}-\frac{1}{1+\alpha}\left(\frac{1}{4}+\ln 2\right), \\
& \lambda_{1}=-\frac{1-\alpha}{\alpha}\left(\frac{\alpha}{2-\alpha}-\frac{1}{2 \alpha}-\frac{1}{4}-\ln 2\right) .
\end{aligned}
$$

Notice that the first term of the above expansion represents the known solution for the case of a semi-infinite fracture in plane-strain, with $\hat{\Omega} \sim \hat{\xi}^{\alpha}[20]$. Notice also that if we return to the original scale, we obtain

$$
\frac{\pi}{\alpha A} I_{1}^{\text {in }}(\xi ; \beta) \sim \beta \pi \cot \pi \alpha(1-\xi)^{\alpha-1}+\beta^{\alpha} \lambda_{0}+\beta^{\alpha-1} \lambda_{1}(1-\xi), \quad \beta^{-1}(1-\xi) \ll 1,
$$

which indicates that the singular (first) term is actually affected by $\beta$.

The evaluation of the integral $I_{2}^{\text {in }}$ on the other hand, does not require the use of re-scaling. Indeed, we can express $I_{2}^{\text {in }}$ directly as

$$
I_{2}^{\text {in }}(\xi ; \beta)=\frac{1}{2} \Omega(\xi-\beta)+\frac{1}{8} \beta^{2} I_{21}^{\text {in }}(\xi ; \beta)-\frac{3}{128} \beta^{4} I_{22}^{\text {in }}(\xi ; \beta),
$$

where $I_{21}^{\text {in }}$ and $I_{22}^{\text {in }}$ are in this case defined as

$$
I_{21}^{\text {in }}=\int_{-1}^{\xi-\beta} \frac{\Omega^{\prime}\left(\xi_{o}\right)}{\left(\xi_{o}-\xi\right)^{2}} \mathrm{~d} \xi_{o}, \quad I_{22}^{\text {in }}=\int_{-1}^{\xi-\beta} \frac{\Omega^{\prime}\left(\xi_{o}\right)}{\left(\xi_{o}-\xi\right)^{4}} \mathrm{~d} \xi_{o} .
$$

For $I_{21}^{\text {in }}$, we divide the integration interval in the following form

$$
\begin{aligned}
I_{21}^{\text {in }}(\xi ; \beta)= & \alpha A \int_{-1}^{\xi-\delta_{1}} \frac{\left(1+\xi_{o}\right)^{\alpha-1}}{\left(\xi_{o}-\xi\right)^{2}} \mathrm{~d} \xi_{o} \\
& +\int_{\xi-\delta_{1}}^{\xi-\delta_{2}} \frac{\Omega^{\prime}\left(\xi_{o}\right)}{\left(\xi_{o}-\xi\right)^{2}} \mathrm{~d} \xi_{o}-\alpha A \int_{\xi-\delta_{2}}^{\xi-\beta} \frac{\left(1-\xi_{o}\right)^{\alpha-1}}{\left(\xi_{o}-\xi\right)^{2}} \mathrm{~d} \xi_{o} .
\end{aligned}
$$

The first integral is identical to (26a), which implies that:

$$
\int_{-1}^{\xi-\delta_{1}} \frac{\left(1+\xi_{o}\right)^{\alpha-1}}{\left(\xi_{o}-\xi\right)^{2}} \mathrm{~d} \xi_{o} \sim \phi(\alpha)\left(1+\xi_{o}\right)^{\alpha-2}+\frac{1}{\alpha A} g_{11}\left(\xi ; \alpha, \delta_{1}\right)+O\left(\epsilon_{1}\right)
$$


with $\phi$ as defined in (25). For the second integral, we have

$$
\int_{\xi-\delta_{1}}^{\xi-\delta_{2}} \frac{\Omega^{\prime}\left(\xi_{o}\right)}{\left(\xi_{o}-\xi\right)^{2}} \mathrm{~d} \xi_{o}=-g_{11}\left(\xi ; \alpha, \delta_{1}\right)+g_{12}\left(\xi ; \alpha, \delta_{2}\right)
$$

The third integral in (41) has to be carefully evaluated. If we "zoom" into the right tip region, the following picture appears:

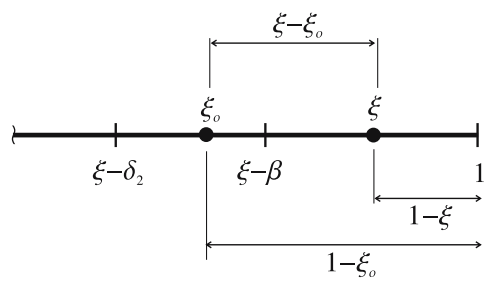

which means that for $\xi$ in the inner region, we have that $1-\xi \ll \xi-\xi_{o}$. We can write

$$
\frac{1}{\left(\xi_{o}-\xi\right)^{2}} \equiv \frac{1}{\left(1-\xi_{o}\right)^{2}\left[1+\left(\frac{1-\xi}{1-\xi_{o}}\right)\right]^{2}}
$$

and perform the following expansion

$$
\frac{1}{\left[1+\left(\frac{1-\xi}{1-\xi_{o}}\right)\right]^{2}} \sim 1-2\left(\frac{1-\xi}{1-\xi_{o}}\right)+3\left(\frac{1-\xi}{1-\xi_{o}}\right)^{2}+O\left[\left(\frac{1-\xi}{1-\xi_{o}}\right)^{3}\right], \quad \frac{1-\xi}{1-\xi_{o}} \ll 1
$$

to obtain

$$
\begin{aligned}
\int_{\xi-\delta_{2}}^{\xi-\beta} & \frac{\left(1-\xi_{o}\right)^{\alpha-1}}{\left(\xi_{o}-\xi\right)^{2}} \mathrm{~d} \xi_{o} \sim-\frac{1}{\alpha-2}(1-\xi+\beta)^{\alpha-2} \\
& +\frac{2}{\alpha-3}(1-\xi)(1-\xi+\beta)^{\alpha-3}+\ldots+\frac{1}{\alpha A} g_{12}\left(\xi ; \alpha, \delta_{2}\right) .
\end{aligned}
$$

However, we also know that $1-\xi<\beta \ll 1$. Hence, we can perform another expansion of the above result for small $1-\xi$, to obtain

$$
\int_{\xi-\delta_{2}}^{\xi-\beta} \frac{\left(1-\xi_{o}\right)^{\alpha-1}}{\left(\xi_{o}-\xi\right)^{2}} \mathrm{~d} \xi_{o} \sim-\frac{\beta^{\alpha-2}}{\alpha-2}-\beta^{\alpha-3}\left(\frac{\alpha-5}{\alpha-3}\right)(1-\xi)+\ldots+\frac{1}{\alpha A} g_{12}\left(\xi ; \alpha, \delta_{2}\right) .
$$

Combination of the above results yields

$$
I_{21}^{\text {in }}(\xi ; \beta)=\alpha A\left[\phi(\alpha)\left(1+\xi_{o}\right)^{\alpha-1}+\beta^{\alpha-2}\left(\frac{1}{\alpha-2}\right)+\beta^{\alpha-3}\left(\frac{\alpha-5}{\alpha-3}\right)(1-\xi)\right] .
$$

The procedure to follow for $I_{22}^{\text {in }}$ is similar. We propose

$$
\begin{aligned}
I_{22}^{\text {in }}(\xi ; \beta)= & \alpha A \int_{-1}^{\xi-\delta_{1}} \frac{\left(1+\xi_{o}\right)^{\alpha-1}}{\left(\xi_{o}-\xi\right)^{4}} \mathrm{~d} \xi_{o} \\
& +\int_{\xi-\delta_{1}}^{\xi-\delta_{2}} \frac{\Omega^{\prime}\left(\xi_{o}\right)}{\left(\xi_{o}-\xi\right)^{4}} \mathrm{~d} \xi_{o}-\alpha A \int_{\xi-\delta_{2}}^{\xi-\beta} \frac{\left(1-\xi_{o}\right)^{\alpha-1}}{\left(\xi_{o}-\xi\right)^{4}} \mathrm{~d} \xi_{o},
\end{aligned}
$$


from which we obtain

$$
\begin{gathered}
\int_{-1}^{\xi-\delta_{1}} \frac{\left(1+\xi_{o}\right)^{\alpha-1}}{\left(\xi_{o}-\xi\right)^{4}} \mathrm{~d} \xi_{o} \sim f_{21}(\xi ; \alpha)+\frac{1}{\alpha A} g_{21}\left(\xi ; \alpha, \delta_{1}\right)+O\left(\epsilon_{1}\right), \\
\int_{\xi-\delta_{1}}^{\xi-\delta_{2}} \frac{\Omega^{\prime}\left(\xi_{o}\right)}{\left(\xi_{o}-\xi\right)^{4}} \mathrm{~d} \xi_{o}=-g_{21}\left(\xi ; \alpha, \delta_{1}\right)+g_{22}\left(\xi ; \alpha, \delta_{2}\right),
\end{gathered}
$$

where we leave $f_{21}$ undefined. To evaluate the third integral in (47), we use

$$
\frac{1}{\left(\xi_{o}-\xi\right)^{4}} \equiv \frac{1}{\left(1-\xi_{o}\right)^{4}\left[1+\left(\frac{1-\xi}{1-\xi_{o}}\right)\right]^{4}}
$$

and

$$
\frac{1}{\left[1+\left(\frac{1-\xi}{1-\xi_{o}}\right)\right]^{4}} \sim 1-4\left(\frac{1-\xi}{1-\xi_{o}}\right)+10\left(\frac{1-\xi}{1-\xi_{o}}\right)^{2}+O\left[\left(\frac{1-\xi}{1-\xi_{o}}\right)^{3}\right], \quad \frac{1-\xi}{1-\xi_{o}} \ll 1,
$$

to obtain

$$
\begin{aligned}
\int_{\xi-\delta_{2}}^{\xi-\beta} & \frac{\left(1-\xi_{o}\right)^{\alpha-1}}{\left(\xi_{o}-\xi\right)^{4}} \mathrm{~d} \xi_{o} \sim-\frac{1}{\alpha-4}(1-\xi+\beta)^{\alpha-4} \\
& +\frac{4}{\alpha-5}(1-\xi)(1-\xi+\beta)^{\alpha-5}+\ldots+\frac{1}{\alpha A} g_{22}\left(\xi ; \alpha, \delta_{2}\right)
\end{aligned}
$$

Again, we perform an expansion for small $1-\xi<\beta$, to obtain

$$
\int_{\xi-\delta_{2}}^{\xi-\beta} \frac{\left(1-\xi_{o}\right)^{\alpha-1}}{\left(\xi_{o}-\xi\right)^{4}} \mathrm{~d} \xi_{o} \sim-\frac{\beta^{\alpha-4}}{\alpha-4}-\beta^{\alpha-5}\left(\frac{\alpha-9}{\alpha-5}\right)(1-\xi)+\ldots+\frac{1}{\alpha A} g_{22}\left(\xi ; \alpha, \delta_{2}\right) .
$$

Combination of the above results yields

$$
I_{22}^{\text {in }}(\xi ; \beta)=\alpha A\left[f_{21}(\xi ; \alpha)+\beta^{\alpha-4}\left(\frac{1}{\alpha-4}\right)+\beta^{\alpha-5}\left(\frac{\alpha-9}{\alpha-5}\right)(1-\xi)\right] .
$$

Considering the definition of $I_{2}^{\text {in }}$ given by (39), and making use of the expansion:

$$
\begin{aligned}
\Omega(\xi-\beta)= & A(1-\xi+\beta)^{\alpha} \sim A\left[\beta^{\alpha}+\alpha \beta^{\alpha-1}(1-\xi)\right] \\
& +O\left[\beta^{\alpha-2}(1-\xi)^{2}\right], \quad 1-\xi \ll \beta,
\end{aligned}
$$

we obtain

$$
\begin{aligned}
I_{2}^{\text {in }}(\xi ; \beta)= & \alpha A \beta^{2} \phi(\alpha)(1+\xi)^{\alpha-2}+A \beta^{\alpha}\left[\frac{1}{2}+\frac{\alpha}{8(\alpha-2)}-\frac{3 \alpha}{128(\alpha-4)}\right] \\
& +\alpha A \beta^{\alpha-1}\left[\frac{1}{2}+\frac{1}{8}\left(\frac{\alpha-5}{\alpha-3}\right)-\frac{3}{128}\left(\frac{\alpha-9}{\alpha-5}\right)\right](1-\xi),
\end{aligned}
$$

where we have truncated terms of order higher than $(1-\xi)^{2}$. 
Combining (38) and (52) we obtain the inner local expansion of the boundary integral operator in the limit $\beta^{-1}(1-\xi) \rightarrow 0$ :

$$
\Pi^{\text {in }}(\xi ; \beta)=\alpha A \beta \cot \pi \alpha(1-\xi)^{\alpha-1}+\beta^{\alpha} \mu_{0}+\beta^{\alpha-1} \mu_{1}(1-\xi),
$$

where the coefficients $\mu_{0}$ and $\mu_{1}$ are defined as

$$
\begin{aligned}
& \mu_{0}=\alpha A\left[\frac{\lambda_{0}}{\pi}+\frac{1}{2 \alpha}+\frac{1}{8(\alpha-2)}-\frac{3}{128(\alpha-4)}\right] \\
& \mu_{1}=\alpha A\left[\frac{\lambda_{1}}{\pi}+\frac{1}{2}+\frac{1}{8}\left(\frac{\alpha-5}{\alpha-3}\right)-\frac{3}{128}\left(\frac{\alpha-9}{\alpha-5}\right)\right] .
\end{aligned}
$$

Equating $\Pi^{\text {in }}$ to the leading order term in the expansion demonstrates that the action of the elasticity integral operator is in this limit the same as the Finite Hilbert Transform associated with a state of plane strain. In this limit, the state of plane strain prevails because the receiving point is sufficiently close to the tip $x=\ell$ that variation of the solution in the $y$ direction becomes insignificant compared to that in the $x$ direction. This result is important as it will enable the appropriate stress singularities to be incorporated into the model in order to treat the propagation of the fracture in regime in which the toughness is not negligible.

Fig. 6 Plot of the dimensionless pressure $\Pi$ calculated numerically from (9) (dots) versus normalized distance from the tip $(1-\xi) / \beta$. Comparison between $\Pi$, the inner expansion (53) (solid line), the dimensionless fracture opening $\Omega$ (dashed line), and the first term of the expansion (dotted line). Results were obtained using the test function $\Omega=\left(1-\xi^{2}\right)^{2 / 3}$ and $\beta=0.01$
$\Pi, \Omega$

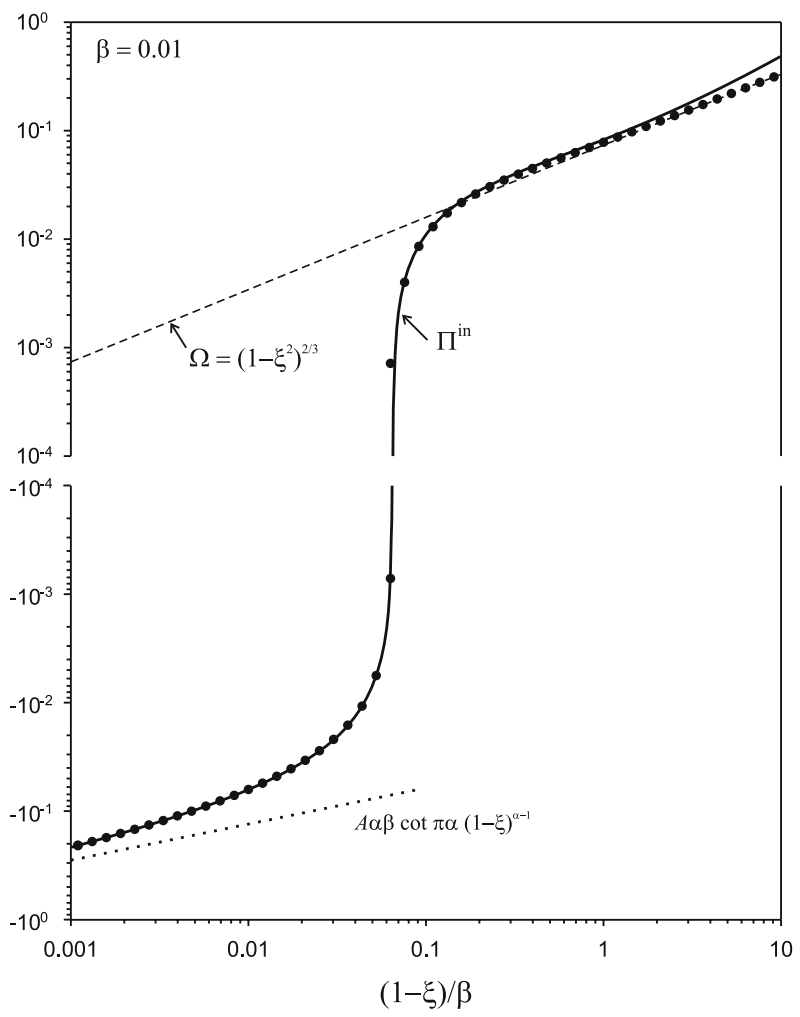


A plot of the inner expansion is shown in Fig. 6. Again, this plot was obtained by using a "test function" $\Omega=\left(1-\xi^{2}\right)^{2 / 3}$ and $\beta=0.01$. Notice that the inner expansion is valid on the interval $1-\beta<\xi<1$. We have also plotted the first term of the inner expansion $\alpha A \beta \cot \pi \alpha(1-\xi)^{\alpha-1}$ (dotted line), which corresponds to the "planestrain" pressure singularity [20]. It is evident that the solution starts to converge towards this term for $(1-\xi) \lesssim 10^{-3} \beta$.

\subsection{Intermediate Expansions}

The outer and inner expansions have been obtained under the assumption that $|\xi| \ll 1-\beta$ and $|\xi| \gg 1-\beta$ respectively. From the results presented in Fig. 5 it is evident that the zeroth order outer expansion $\Pi=\Omega$ is valid in the region $1-\beta<$ $\xi<100 \beta$, while the higher order outer expansion (32) is only valid on the smaller region $1-3 \beta<\xi<100 \beta$. Thus in order to achieve a higher order representation of the solution we require an approximate characterization of the integral operator (9) in the intermediate interval $1-3 \beta<\xi<1-\beta$. We subdivide the intermediate interval into two subintervals $1-3 \beta<\xi<1-2 \beta$ and $1-2 \beta<\xi<1-\beta$ and on each of these we give distinct approximations to the integral operator defined in (9).

Let us introduce the notation

$$
\Omega(\xi)= \begin{cases}\Omega^{\mathrm{OUT}}(\xi),-1<\xi<1-3 \beta \\ \Omega^{\mathrm{Int}}(\xi), & 1-3 \beta<\xi<1-\beta \\ \Omega^{\text {in }}(\xi), & 1-\beta<\xi<1 .\end{cases}
$$

It is assumed for this section that $\Omega^{\mathrm{OUT}}(\xi)$ and $\Omega^{\text {in }}(\xi)$ are known functions since they would have been determined by making use of the local equations (32) and (53).

For values of $\xi$ in the range $1-3 \beta<\xi<1-2 \beta$ the following picture applies:

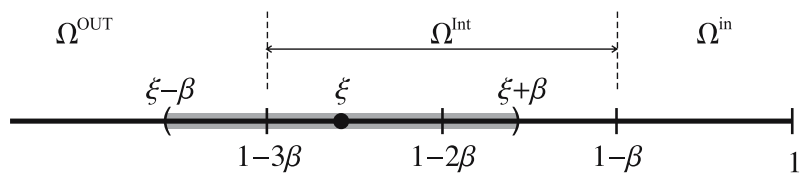

and hence, the expression (16) for $\Pi(\xi ; \beta)$ can be approximated as follows:

$$
\begin{aligned}
\Pi_{\mathrm{I}}^{\text {Int }}(\xi ; \beta)= & \Omega^{\mathrm{Int}}(\xi)+\beta^{2}\left[\frac{1}{2}-\frac{1}{\pi}\left(\frac{41}{18}+\frac{2}{3} \ln 2\right)\right] \frac{\mathrm{d}^{2}}{\mathrm{~d} \xi^{2}} \Omega^{\text {Int }}(\xi) \\
& +\beta^{4}\left(\frac{1}{24}-\frac{83}{450 \pi}\right) \frac{\mathrm{d}^{4}}{\mathrm{~d} \xi^{4}} \Omega^{\mathrm{Int}}(\xi)+\ldots-\beta^{2} \int_{\xi+\beta}^{1-\beta} \frac{\mathrm{d}}{\mathrm{d} \xi_{o}} \Omega^{\text {Int }}\left(\xi_{o}\right) G_{2}\left(\xi, \xi_{o}\right) \mathrm{d} \xi_{o} \\
& +\beta^{2} \int_{-1}^{\xi-\beta} \frac{\mathrm{d}}{\mathrm{d} \xi_{o}} \Omega^{\mathrm{OUT}}\left(\xi_{o}\right) G_{2}\left(\xi, \xi_{o}\right) \mathrm{d} \xi_{o}-\beta^{2} \int_{1-\beta}^{1} \frac{\mathrm{d}}{\mathrm{d} \xi_{o}} \Omega^{\mathrm{in}}\left(\xi_{o}\right) G_{2}\left(\xi, \xi_{o}\right) \mathrm{d} \xi_{o} .
\end{aligned}
$$

We observe that the series expansion terms result from the expansion of the first term on the right of (16) combined with an approximation of the Cauchy Principal Value integral $f_{\xi-\beta}^{\xi+\beta}$ in which a power series expansion of $\Omega$ is assumed. The implicit assumption in this expansion is that $\Omega$ is sufficiently smooth over the transition point $\xi=1-3 \beta$ for the expansion to be valid. The first integral on the right side of (54) involves the unknown function $\Omega^{\text {Int }}$ while the last two integrals involve the functions 
$\Omega^{\text {OUT }}$ and $\Omega^{\text {in }}$ that are both assumed to be known. In the analysis presented in Sections 4.2 and 4.3 the outer and the inner expansions reduce the integral operator to local operators. However, in this case the Fredholm integral operator of the first kind is reduced to an integro-differential operator of the second kind in which the integral term as well as the differential terms involve small perturbations. A Neumann type series can therefore be expected to yield a convergent solution. The remaining integrals in (54) involve quadratures of the known functions $\Omega^{\text {OUT }}$ and $\Omega^{\text {in }}$ and can be regarded as forcing terms. In the case that $\Omega^{\text {in }}$ is in the form of a power law (which is to be expected given the inner expansion) an explicit formula for the last integral in (54) is given in Appendix C.

For values of $\xi$ in the range $1-2 \beta<\xi<1-\beta$, on the other hand, we have:

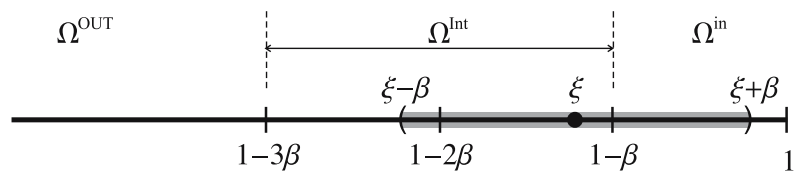

and the appropriate expression for $\Pi(\xi ; \beta)$ is as follows:

$$
\begin{aligned}
\Pi_{\mathrm{II}}^{\mathrm{Int}}(\xi ; \beta)= & \frac{1}{2}\left[\Omega^{\mathrm{Int}}(\xi-\beta)+\Omega^{\text {in }}(\xi+\beta)\right]+ \\
& +\beta^{2} \int_{1-3 \beta}^{\xi-\beta} \frac{\mathrm{d}}{\mathrm{d} \xi_{o}} \Omega^{\text {Int }}\left(\xi_{o}\right) G_{2}\left(\xi, \xi_{o}\right) \mathrm{d} \xi_{o}+f_{\xi-\beta}^{\xi+\beta} \Omega^{\prime}\left(\xi_{o}\right) G_{1}\left(\xi, \xi_{o}\right) \mathrm{d} \xi_{o} \\
& +\beta^{2} \int_{-1}^{1-3 \beta} \frac{\mathrm{d}}{\mathrm{d} \xi_{o}} \Omega^{\mathrm{OUT}}\left(\xi_{o}\right) G_{2}\left(\xi, \xi_{o}\right) \mathrm{d} \xi_{o}-\beta^{2} \int_{\xi+\beta}^{1} \frac{\mathrm{d}}{\mathrm{d} \xi_{o}} \Omega^{\text {in }}\left(\xi_{o}\right) G_{2}\left(\xi, \xi_{o}\right) \mathrm{d} \xi_{o} .
\end{aligned}
$$

Here the Cauchy Principal Value integral can be further decomposed as follows:

$$
\begin{aligned}
\int_{\xi-\beta}^{\xi+\beta} \Omega^{\prime}\left(\xi_{o}\right) G_{1}\left(\xi, \xi_{o}\right) \mathrm{d} \xi_{o}= & f_{\xi-\beta}^{\xi+\beta}\left(\Omega^{\prime}\left(\xi_{o}\right)-\Omega^{\prime}(\xi)\right) G_{1}\left(\xi, \xi_{o}\right) \mathrm{d} \xi_{o} \\
& +\Omega^{\prime}(\xi) f_{\xi-\beta}^{\xi+\beta} G_{1}\left(\xi, \xi_{o}\right) \mathrm{d} \xi_{o} \\
= & f_{\xi-\beta}^{\xi+\beta}\left(\Omega^{\prime}\left(\xi_{o}\right)-\Omega^{\prime}(\xi)\right) G_{1}\left(\xi, \xi_{o}\right) \mathrm{d} \xi_{o}
\end{aligned}
$$

since the kernel function $G_{1}\left(\xi, \xi_{o}\right)$ is an anti-symmetric function of $\xi_{o}$ about the point $\xi$. Thus for $1-2 \beta<\xi<1-\beta$ we have:

$$
\begin{aligned}
\int_{\xi-\beta}^{\xi+\beta} \Omega^{\prime}\left(\xi_{o}\right) G_{1}\left(\xi, \xi_{o}\right) \mathrm{d} \xi_{o}= & \int_{\xi-\beta}^{1-\beta}\left[\frac{\mathrm{d}}{\mathrm{d} \xi_{o}} \Omega^{\text {Int }}\left(\xi_{o}\right)-\frac{\mathrm{d}}{\mathrm{d} \xi} \Omega^{\text {Int }}(\xi)\right] G_{1}\left(\xi, \xi_{o}\right) \mathrm{d} \xi_{o} \\
& +\int_{1-\beta}^{\xi+\beta}\left[\frac{\mathrm{d}}{\mathrm{d} \xi_{o}} \Omega^{\text {in }}\left(\xi_{o}\right)-\frac{\mathrm{d}}{\mathrm{d} \xi} \Omega^{\text {Int }}(\xi)\right] G_{1}\left(\xi, \xi_{o}\right) \mathrm{d} \xi_{o} .
\end{aligned}
$$

Provided $\Omega^{\text {Int }}$ is sufficiently smooth then the first integral is regular, while an explicit expression for the second integral is given in Appendix C for the case in which $\Omega^{\text {in }}$ is given by a power law. We observe that provided the first derivatives of $\Omega^{\text {Int }}$ and $\Omega^{\text {in }}$ are continuous at $\xi=1-\beta$ then the second integral is also finite as $\xi \rightarrow 1-\beta$. 
Fig. 7 Plot of the dimensionless pressure $\Pi$ calculated numerically from (9) (large dots) versus normalized distance from the tip $(1-\xi) / \beta$. Comparison between $\Pi$, the intermediate expansions $\Pi_{\mathrm{I}}^{\mathrm{Int}}$ (54) (dashed line) and $\Pi_{\mathrm{II}}^{\mathrm{Int}}(55)$ (solid line), and the dimensionless fracture opening $\Omega \simeq \Pi$ (dotted line). Results were obtained using the test function $\Omega=\left(1-\xi^{2}\right)^{2 / 3}$ and $\beta=0.01$

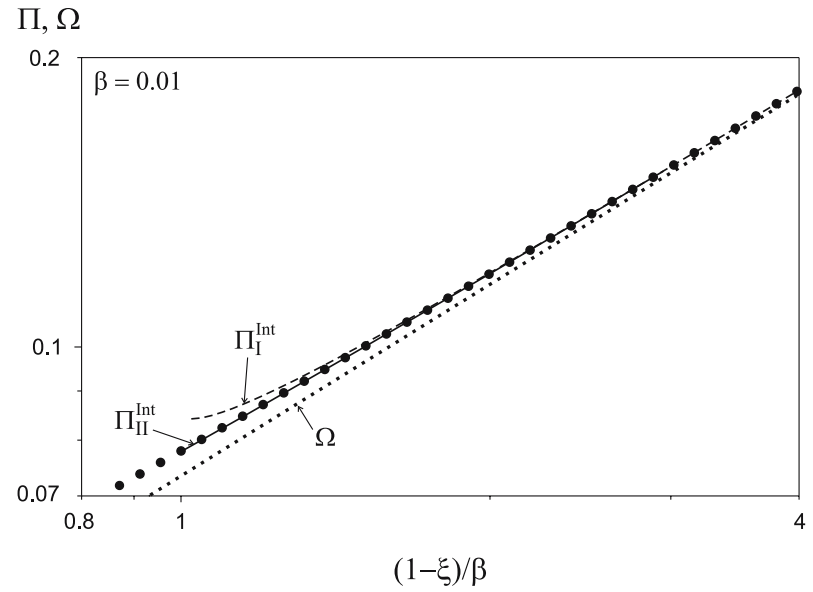

In Fig. 7 we compare the evaluation of $\Pi^{\mathrm{Int}}(\xi ; \beta)$ using the approximate operators given by (54) (dashed) and (55) (solid) with a numerical evaluation of the elasticity integral operator (9) (solid circles) as well as the purely local approximation $\Pi^{\text {Int }}(\xi ; \beta) \simeq \Omega^{\text {Int }}(\xi)$ (dotted) for points $\xi$ in the vicinity of the intermediate region $1-3 \beta<\xi<1-\beta$. For the purposes of this comparison, we have used the same test function as was used in the previous sections on the outer and inner expansions. The values of $\Pi^{\mathrm{Int}}(\xi ; \beta)$, generated by the intermediate approximate operators, show good agreement with the values from the elasticity operator (9) in the particular regions in which they are valid and the deviation of the purely local approximation in this region can be clearly seen. The deviation of the simpler intermediate expansion (54) from the elasticity operator in the region $1-2 \beta<\xi<1-\beta$ clearly indicates the necessity of the more complex expansion (55). In Fig. 8 we compare the values of $\Pi^{\text {Int }}(\xi ; \beta)$ obtained using the outer, inner, and intermediate expansions with those obtained from the numerical evaluation of the elasticity integral operator (9) and the purely local approximation $\Pi^{\operatorname{Int}}(\xi ; \beta) \simeq \Omega^{\text {Int }}(\xi)$ on an interval that straddles

Fig. 8 Plot of the dimensionless pressure $\Pi$ calculated numerically from (9) (large dots) versus normalized distance from the tip $(1-\xi) / \beta$. Comparison between $\Pi$, the inner (small dashes), the intermediate expansions $\Pi_{\mathrm{I}}^{\mathrm{Int}}$ (54) (dashed line) and $\Pi_{\mathrm{II}}^{\mathrm{Int}}(55)$ (solid line), the outer (dash-dots)

expansion, and the

dimensionless fracture opening $\Omega \simeq \Pi$ (dotted line). Results were obtained using the test function

$\Omega=\left(1-\xi^{2}\right)^{2 / 3}$ and $\beta=0.01$

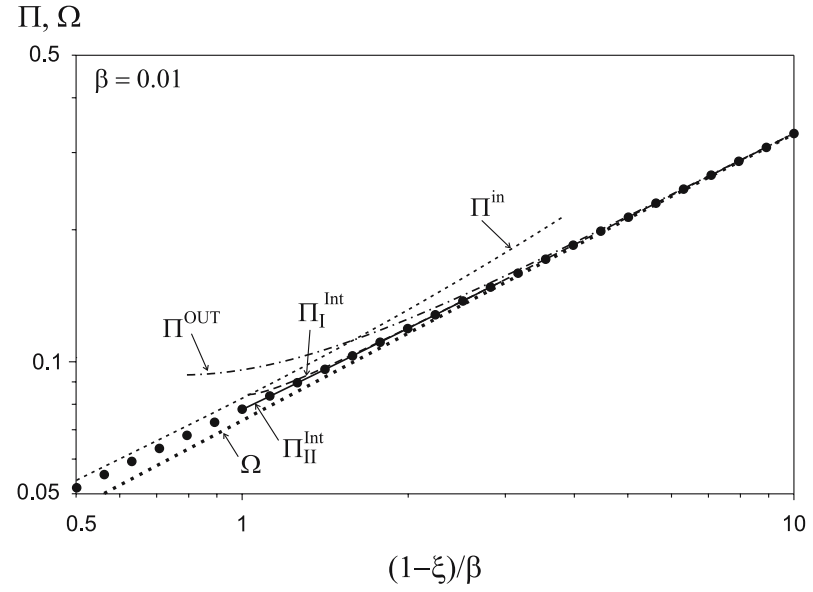


the intermediate region $1-3 \beta<\xi<1-\beta$. Each of these expansions accurately characterizes the action of the elasticity operator (9) in their respective intervals of validity. The error in the purely local approximation $\Pi^{\operatorname{Int}}(\xi ; \beta) \simeq \Omega^{\operatorname{Int}}(\xi)$ can be clearly seen.

\section{Conclusions}

The main conclusions of this analysis are the following:

1. A novel consistent formulation of a non-local elasticity equation for finger-like hydraulic fractures has been introduced. This singular integral equation with a Cauchy type kernel enables the nonlocal effects of width variations over the length of a finger-like hydraulic fracture to be incorporated. This model promises to remedy some serious shortcomings of the classical PKN model, which is restricted to hydraulic fractures for which the fluid pressure approaches zero at the tip.

2. An asymptotic analysis of the non-local elasticity equation in the outer region $\beta \ll 1-\xi<1$, reveals that the leading order action of the elasticity operator equates the dimensionless pressure to the dimensionless width. This result establishes, in a more rigorous way, the "classical" local elasticity equation of the PKN model when the aspect ratio of the fracture $\beta=H / \ell$ becomes vanishingly small. We have also obtained the correction terms to the PKN elasticity equation as a function of $\beta$. These correction terms involve higher width derivatives that account for the nonlocal effects in the elasticity integral equation due to variations in the width.

3. An asymptotic analysis of the proposed elasticity equation in the near-tip region $1-\xi<\beta \ll 1$, reveals that the action of the elasticity integral operator is, in this limit, the same as the Finite Hilbert Transform associated with a state of plane strain. Thus we have established the existence of a relatively small region at the fracture tip, in which fracture opening and pressure are related as in a planestrain fracture. As a consequence, if we assume a fracture tip shape of the form $(1 \mp \xi)^{\alpha}$, then pressure singularities of the form $(1 \mp \xi)^{\alpha-1}$ are observed near the tip, as in the case of a plane-strain fracture [20]. This result is important as it will enable the appropriate stress singularities to be incorporated into the model, for example, in order to treat the propagation of the fracture in regime in which the toughness is not negligible.

4. An asymptotic analysis of the non-local elasticity equation in an intermediate region $1-3 \beta<\xi<1-\beta$ yields two distinct expansions in each of which the action of the Fredholm integral operator of the first kind is reduced to a second kind integral equation in which the integral operator terms appear as small perturbations. These reduced equations will therefore lead to convergent Neumann series.

5. A test function is used to provide numerical comparisons between the action of the non-local elasticity operator and that of the outer, the inner, and intermediate expansions in their various regains of validity. These tests verify the approximate characterization of the action of the elasticity integral operator by simpler operators which are either local or are second kind operators with convergent Neumann series. 
6. The next step in this ongoing project consists of coupling the new elasticity equation with the rest of the equations of the PKN model (namely, the lubrication and volume balance equations). We expect that this coupling will yield the correct shape of the fracture at the near-tip region (i.e., the exponent $\alpha$ ). An additional layer of difficulty that can be foreseen is the fact that, once we start considering a propagating fracture, the small parameter used for our asymptotic analysis (the number $\beta$ ) will become a function of time.

7. We also postulate that, due to the fact that the outer solution should correspond to the "classical" PKN solution (which predicts a regular pressure at the tip), and that the resultant near-tip or inner behavior of the pressure at the tip is singular, a boundary layer (the thickness of which has yet to be determined) should form at the tip region. The thickness of this boundary layer should determine the relevance of the pressure singularity in the propagation of the fracture.

8. This new formulation of the elasticity equation opens the possibility of adding other parameters to the PKN model, such as rock toughness, leak-off, fluid lag, and distance to a free-surface, in a rigorous manner.

Acknowledgements Anthony Peirce acknowledges the support of the NSERC discovery grants program, and José Adachi thanks Schlumberger for permission to publish. Both authors would like to thank Profs. Emmanuel Detournay (University of Minnesota) and Dmitry Garagash (Clarkson University) for the benefits of many discussions. We are also indebted to Dr. Sarah Mitchell (currently at Cape Town University) for thoroughly checking the calculations and for some useful suggestions that were incorporated into the text.

\section{Appendix A: Calculation of Integrals for Outer Expansion}

We start with $I_{1}^{\mathrm{OUT}}(\xi ; \beta)$, as defined in $(19 \mathrm{a})$, which we repeat for convenience

$$
I_{1}^{\mathrm{OUT}}(\xi ; \beta)=f_{\xi-\beta}^{\xi+\beta} \Omega^{\prime}\left(\xi_{o}\right) G_{1}\left(\xi, \xi_{o}\right) \mathrm{d} \xi_{o},
$$

with the kernel $G_{1}$ being defined in (17a). Recall that we are assuming that $\Omega$ (the dimensionless fracture opening) is an analytic, symmetric (even), positive function in $\xi \in[-1,1]$, with $\Omega(\xi= \pm 1)=0$. Let us define

$$
\begin{aligned}
& I_{11}^{\mathrm{OUT}}=-\frac{\beta}{\pi} f_{\xi-\beta}^{\xi+\beta} \Omega^{\prime}\left(\xi_{o}\right) \frac{\mathrm{d} \xi_{o}}{\xi_{o}-\xi}, \\
& I_{12}^{\text {OUT }}=\frac{1}{2 \pi \beta} \int_{\xi-\beta}^{\xi+\beta} \Omega^{\prime}\left(\xi_{o}\right)\left(\xi_{o}-\xi\right) \ln \frac{\left|\xi_{o}-\xi\right|}{\beta} \mathrm{d} \xi_{o} \\
& I_{13}^{\text {OUT }}=-\frac{1}{\pi \beta}\left(\frac{1}{4}+\ln 2\right) \int_{\xi-\beta}^{\xi+\beta} \Omega^{\prime}\left(\xi_{o}\right)\left(\xi_{o}-\xi\right) \mathrm{d} \xi_{o} .
\end{aligned}
$$

As we are also assuming that $[\xi-\beta, \xi+\beta] \subset\left[\xi-\delta_{1}, \xi+\delta_{2}\right]$, these integrals can be evaluated by first performing a Taylor's series expansion of $\Omega^{\prime}$ around $\xi_{o}=\xi$

$$
\Omega^{\prime}\left(\xi_{o}\right)=\Omega^{\prime}(\xi)+\left(\xi_{o}-\xi\right) \Omega^{\prime \prime}(\xi)+\frac{1}{2}\left(\xi_{o}-\xi\right)^{2} \Omega^{\prime \prime \prime}(\xi)+\ldots
$$


If we truncate this expansion at the fourth term, and substitute it into the above integrals, we obtain (taking advantage of the symmetry of the integration interval)

$$
\begin{aligned}
& I_{11}^{\mathrm{OUT}}=-\frac{2}{\pi} \beta^{2} \Omega^{\prime \prime}(\xi)-\frac{\beta^{4}}{9 \pi} \Omega^{(4)}(\xi), \\
& I_{12}^{\mathrm{OUT}}=-\frac{\beta^{2}}{9 \pi} \Omega^{\prime \prime}(\xi)-\frac{\beta^{4}}{150 \pi} \Omega^{(4)}(\xi), \\
& I_{13}^{\mathrm{OUT}}=-\frac{2}{3 \pi}\left(\frac{1}{4}+\ln 2\right) \beta^{2} \Omega^{\prime \prime}(\xi)-\frac{\beta^{4}}{15 \pi} \Omega^{(4)}(\xi) .
\end{aligned}
$$

Combination of the above results yields

$$
I_{1}^{\mathrm{OUT}}(\xi ; \beta)=-\frac{\beta^{2}}{\pi}\left(\frac{41}{18}+\frac{2}{3} \ln 2\right) \Omega^{\prime \prime}(\xi)-\frac{\beta^{4}}{\pi}\left(\frac{83}{450}\right) \Omega^{(4)}(\xi) .
$$

\section{Appendix B: Calculation of Integrals for Inner Expansion}

In this appendix, we present the closed form solution for the following integral

$J_{1}(\hat{\xi})=\frac{A \alpha}{\pi} f_{0}^{\hat{\xi}+1} \hat{\xi}_{o}^{\alpha-1}\left[\frac{1}{\hat{\xi}_{o}-\hat{\xi}}-\frac{1}{2}\left(\hat{\xi}_{o}-\hat{\xi}\right) \ln \left|\hat{\xi}_{o}-\hat{\xi}\right|+\left(\frac{1}{4}+\ln 2\right)\left(\hat{\xi}_{o}-\hat{\xi}\right)\right] \mathrm{d} \hat{\xi}_{o}, \hat{\xi}<1$

First, we subdivide $J_{1}$ into the following parts

$$
\begin{aligned}
& J_{11}=\int_{0}^{\hat{\xi}+1} \hat{\xi}_{o}^{\alpha-1} \frac{\mathrm{d} \hat{\xi}_{o}}{\hat{\xi}_{o}-\hat{\xi}}, \\
& J_{12}=\int_{0}^{\hat{\xi}+1} \hat{\xi}_{o}^{\alpha-1}\left(\hat{\xi}_{o}-\hat{\xi}\right) \ln \left|\hat{\xi}_{o}-\hat{\xi}\right| \mathrm{d} \hat{\xi}_{o} \\
& J_{13}=\int_{0}^{\hat{\xi}+1} \hat{\xi}_{o}^{\alpha-1}\left(\hat{\xi}_{o}-\hat{\xi}\right) \mathrm{d} \hat{\xi}_{o} .
\end{aligned}
$$

Closed-form expressions for each of these integrals are as follows:

$$
\begin{aligned}
& J_{11}=\hat{\xi}^{\alpha-1}\left[\pi \cot \pi \alpha+\mathrm{B}_{\frac{\hat{\xi}}{1+\hat{\xi}}}(1-\alpha, 0)\right], \\
& J_{12}=-\frac{1}{\alpha(\alpha+1)}\left\{\hat{\xi}^{\alpha+1}\left[\pi \cot \pi \alpha+\mathrm{B}_{\frac{\hat{\xi}}{1+\hat{\xi}}}(1-\alpha, 0)\right]+(1+\hat{\xi})^{\alpha}\left[\frac{\alpha^{2}-\hat{\xi}(1+\alpha(1-\alpha))}{\alpha(\alpha+1)}\right]\right\}, \\
& J_{13}=\frac{1}{\alpha(\alpha+1)}(\alpha-\hat{\xi})(1+\hat{\xi})^{\alpha} .
\end{aligned}
$$

where $\mathrm{B}_{\phi(\xi)}(\cdot, \cdot)$ is the incomplete beta function [18]. It is worth mentioning here that $\mathrm{B}_{\frac{\hat{\xi}}{1+\hat{\xi}}}(1-\alpha, 0)$ is regular for $\hat{\xi} \geq 0$, which means that the only singularity in (63) 
is given by the term proportional to $\hat{\xi}^{\alpha-1}$. To obtain the asymptotic expansions of (63) for $\hat{\xi} \ll 1$, we will make use of the following expansion for the incomplete beta function

$$
\mathrm{B}_{\frac{\hat{\xi}}{1+\xi}}(1-\alpha, 0) \sim \frac{1}{1-\alpha} \hat{\xi}^{1-\alpha}-\left(\frac{1-\alpha}{2-\alpha}\right) \hat{\xi}^{2-\alpha}+\frac{1}{2} \frac{(2-\alpha)(1-\alpha)}{(3-\alpha)} \hat{\xi}^{3-\alpha}+O\left(\hat{\xi}^{4-\alpha}\right), \hat{\xi} \ll 1 .
$$

In this form, we obtain,

$$
\begin{aligned}
& J_{11} \sim \pi \cot \pi \alpha \hat{\xi}^{\alpha-1}+\frac{1}{1-\alpha}-\left(\frac{1-\alpha}{2-\alpha}\right) \hat{\xi}+O\left(\hat{\xi}^{2}\right), \\
& J_{12} \sim \frac{1}{(1+\alpha)^{2}}+\frac{1-\alpha}{\alpha^{2}} \hat{\xi}-\frac{\pi \cot \pi \alpha}{\alpha(\alpha+1)} \hat{\xi}^{\alpha+1}+O\left(\hat{\xi}^{2}\right), \\
& J_{13} \sim-\frac{1}{1+\alpha}+\frac{1-\alpha}{\alpha} \hat{\xi}+O\left(\hat{\xi}^{2}\right) .
\end{aligned}
$$

\section{Appendix C: Calculation of Integrals for the Intermediate Expansion}

In this appendix we provide explicit formulae for the evaluation of integrals for the intermediate expansion that involve $\Omega^{\text {in }}$ which is assumed to be a power law.

In order to evaluate $\int_{1-\beta}^{1} \frac{\mathrm{d}}{\mathrm{d} \xi_{o}} \Omega^{\mathrm{in}}\left(\xi_{o}\right) G_{2}\left(\xi, \xi_{o}\right) \mathrm{d} \xi_{o}$ in (54) the following formulae are useful:

$$
\int_{1-\beta}^{1} \frac{\left(1-\xi_{o}\right)^{\alpha-1}}{\left(\xi_{o}-\xi\right)^{2}} \mathrm{~d} \xi_{o}=\frac{\beta^{\alpha}}{\alpha}(1-\xi-\beta)^{-2}{ }_{2} \mathrm{~F}_{1}\left(1,2 ; \alpha+1 ;-\frac{\beta}{1-\xi-\beta}\right),
$$

and

$$
\begin{aligned}
\int_{1-\beta}^{1} \frac{\left(1-\xi_{o}\right)^{\alpha-1}}{\left(\xi_{o}-\xi\right)^{4}} \mathrm{~d} \xi_{o} \\
=\frac{\beta^{\alpha}}{(1-\xi)^{4}}\left\{\frac{1}{\alpha}-\frac{\beta}{6(1-\xi-\beta)^{3}}\left[(\alpha(7-\alpha)-18)(1-\xi)^{2}\right.\right. \\
\\
\quad-\beta(\alpha(13-2 \alpha)-27)(1-\xi)+\beta^{2}(\alpha(6-\alpha)-11)+ \\
\left.\left.\quad-\frac{(1-\alpha)(2-\alpha)(3-\alpha)}{1+\alpha}(1-\xi-\beta)_{2}{ }_{2} \mathrm{~F}_{1}\left(1,1 ; \alpha+2 ;-\frac{\beta}{1-\xi-\beta}\right)\right]\right\}
\end{aligned}
$$

In order to evaluate $\int_{\xi-\beta}^{1-\beta} \frac{\mathrm{d}}{\mathrm{d} \xi_{o}} \Omega^{\text {in }} G_{1}\left(\xi, \xi_{o}\right) \mathrm{d} \xi_{o}$ in (56) the following integrals are required:

$$
\begin{aligned}
I_{11}^{\text {in }} & =\int_{1-\beta}^{\xi+\beta} \frac{\left(1-\xi_{o}\right)^{\alpha-1}}{\xi_{o}-\xi} \mathrm{d} \xi_{o}, \\
I_{12}^{\text {in }} & =\int_{1-\beta}^{\xi+\beta}\left(1-\xi_{o}\right)^{\alpha-1}\left(\xi_{o}-\xi\right) \ln \left|\frac{\xi_{o}-\xi}{\beta}\right| \mathrm{d} \xi_{o}, \\
I_{13}^{\text {in }} & =\int_{1-\beta}^{\xi+\beta}\left(1-\xi_{o}\right)^{\alpha-1}\left(\xi_{o}-\xi\right) \mathrm{d} \xi_{o} .
\end{aligned}
$$


Closed-form solutions for each of these integrals are as follows:

$$
\begin{aligned}
& I_{11}^{\text {in }}=-\frac{1}{\alpha}[-\frac{\beta^{\alpha}}{1-\xi-\beta}{ }_{2} \mathrm{~F}_{1}\left(1,1 ; \alpha+1 ;-\frac{\beta}{1-\xi-\beta}\right) \\
&\left.+\frac{(1-\xi-\beta)^{\alpha}}{\beta}{ }_{2} \mathrm{~F}_{1}\left(1,1 ; \alpha+1 ;-\frac{1-\xi-\beta}{\beta}\right)\right], \\
& I_{12}^{\text {in }}=\frac{1}{\alpha(\alpha+1)}\left\{\beta^{\alpha+1}-(1-\xi-\beta)^{\alpha+1}-(\alpha+1)(1-\xi)^{\alpha}\left[\beta{ }_{3} \mathrm{~F}_{2}\left(1,1,-\alpha ; 2,2 ; \frac{\beta}{1-\xi}\right)\right.\right. \\
&\left.\quad-(1-\xi-\beta){ }_{3} \mathrm{~F}_{2}\left(1,1,-\alpha ; 2,2 ; 1-\frac{\beta}{1-\xi}\right)\right]+ \\
&\left.\quad+\left\{-(1-\xi)^{\alpha+1}+\beta^{\alpha}[(\alpha+1)(1-\xi)-\alpha \beta]\right\} \ln \left(\frac{1-\xi-\beta}{\beta}\right)\right\},
\end{aligned}
$$

$$
I_{13}^{\text {in }}=-\frac{1}{\alpha(\alpha+1)}\left\{(1-\xi-\beta)^{\alpha}(1-\xi+\alpha \beta)+\beta^{\alpha}[\alpha \beta-(\alpha+1)(1-\xi)]\right\},
$$

where ${ }_{3} \mathrm{~F}_{2}(\cdot, \cdot, \cdot ; \cdot, \cdot ; \cdot)$ is the generalized hypergeometric function. Expansion of (69a) for $\beta \ll 1$ yields:

$$
\begin{aligned}
I_{11}^{\text {in }} \sim & -\beta^{\alpha-1}\left[\ln \left(\frac{1-\xi-\beta}{\beta}\right)+\gamma+\psi(\alpha)\right] \\
& +\alpha(1-\alpha) \beta^{\alpha-2}\left[\ln \left(\frac{1-\xi-\beta}{\beta}\right)+\gamma+\psi(\alpha)-1\right](1-\xi-\beta) \\
& +\frac{1}{\alpha \beta}\left[\frac{1-\xi-\beta}{(1+\alpha) \beta}-1\right](1-\xi-\beta)^{\alpha},
\end{aligned}
$$

where $\gamma$ is Euler's constant and $\psi(\cdot)$ is the digamma function. Since $\int_{1-\beta}^{\xi+\beta} \frac{1}{\xi_{o}-\xi} d \xi_{o}=$ $\ln \left(\frac{1-\xi-\beta}{\beta}\right)$ we observe that the singular logarithmic terms in (56) will cancel provided that $\lim _{\xi \rightarrow(1-\beta)^{-}} \frac{\mathrm{d}}{\mathrm{d} \xi} \Omega^{\text {Int }}(\xi)=\frac{\mathrm{d}}{\mathrm{d} \xi} \Omega^{\text {in }}(1-\beta)$.

In order to evaluate $\int_{\xi+\beta}^{1} \frac{\mathrm{d}}{\mathrm{d} \xi} \Omega^{\text {in }}\left(\xi_{o}\right) G_{2}\left(\xi, \xi_{o}\right) \mathrm{d} \xi_{o}$ in (55) the following formulae are useful:

$$
\int_{\xi+\beta}^{1} \frac{\left(1-\xi_{o}\right)^{\alpha-1}}{\left(\xi_{o}-\xi\right)^{2}} \mathrm{~d} \xi_{o}=\frac{(1-\xi-\beta)^{\alpha}}{\beta(1-\xi)}\left[1+\left(\frac{1-\alpha}{\alpha}\right){ }_{2} \mathrm{~F}_{1}\left(1,1 ; \alpha+1 ; 1-\frac{1-\xi}{\beta}\right)\right],
$$

and

$$
\begin{aligned}
\int_{\xi+\beta}^{1} \frac{\left(1-\xi_{o}\right)^{\alpha-1}}{\left(\xi_{o}-\xi\right)^{4}} \mathrm{~d} \xi_{o}= & \frac{1}{3} \beta^{-3}(1-\xi-\beta)^{\alpha-1}-\frac{1}{6} \beta^{-2}(\alpha-1)(1-\xi-\beta)^{\alpha-2}+ \\
& +\frac{1}{6} \beta^{-1}(\alpha-1)(\alpha-2)(1-\xi-\beta)^{\alpha-3}\left[1-{ }_{2} \mathrm{~F}_{1}\left(1,1 ; \alpha-2 ; 1-\frac{1-\xi}{\beta}\right)\right] .
\end{aligned}
$$




\section{References}

1. Lister, J.R.: Buoyancy-driven fluid fracture: the effects of material toughness and of low-viscosity precursors. J. Fluid Mech. 210, 263-280 (1990)

2. Rubin, A.M.: Propagation of magma-filled cracks. Ann. Rev. Earth Planet. Sci. 23, 287-336 (1995)

3. Spence, D.A., Turcotte, D.L.: Magma-driven propagation crack. J. Geophys. Res. 90, 575-580 (1985) Rotterdam

4. Harrison, E., Kieschnick, W.F., McGuire, W.J.: The mechanics of fracture induction and extension. Petroleum Trans. AIME. (201), 252-263 (1954)

5. Gidley, J.L., Holditch, S.A., Nierode, D.E., Veatch, R.W. (eds.): Recent Advances in Hydraulic Fracturing, volume 12 of SPE Monograph Series. Society of Petroleum Engineers, Richardson TX (1989)

6. Valko, P., Economides, M.J.: Hydraulic Fracture Mechanics. Wiley, Chichester, UK (1995)

7. Economides, M.J., Nolte, K.G. (eds.): Reservoir Stimulation. Wiley, Chichester, UK, 3rd edition (2000)

8. Jeffrey, R.G., Mills, K.W.: Hydraulic fracturing applied to inducing longwall coal mine goaf falls. In: Pacific Rocks 2000, pages 423-430. Rotterdam, Balkema (2000)

9. van As, A., Jeffrey, R.G.: Caving induced by hydraulic fracturing at Northparkes mines. In: Pacific Rocks 2000, pages 353-360. Rotterdam, Balkema (2000)

10. Frank, U., Barkley, N.: Remediation of low permeability subsurface formations by fracturing enhancements of soil vapor extraction. J. Hazard. Mater. 40, 191-201 (2005)

11. Murdoch, L.C.: Mechanical analysis of idealized shallow hydraulic fracture. J. Geotech. Geoenviron. 128(6), 488-495 (2002)

12. Murdoch, L.C., Slack, W.W.: Forms of hydraulic fractures in shallow fine-grained formations. J. Geotech. Geoenviron. 128(6), 479-487 (2002)

13. Perkins, T., Kern, L.: Widths of hydraulic fractures. J. Pet. Technol. 222, 937-949 (1961)

14. Nordgren, R.: Propagation of vertical hydraulic fractures. J. Pet. Technol. 253 306-314 (1972)

15. Kemp, L.: Study of Nordgren's equation of hydraulic fracturing. SPE Prod. Eng. 311-314 (Aug. 1990)

16. Hills, D., Kelly, P., Dai, D., Korsunsky, A.: Solution of Crack Problems: The Distributed Dislocation Technique. Kluwer, Dordrecht (1996)

17. Kaya, A., Erdogan, F.: On the solution of integral equations with strongly singular kernels. Quart. Appl. Math. 14, 105-122 (1987)

18. Abramowitz, M., Stegun, I. (eds.): Handbook of Mathematical Functions with Formulas, Graphs, and Mathematical Tables. Dover Publications, New York (1972)

19. Lebedev, N.: Special Functions and their Applications. Dover Publications Inc., New York (1972. Transl. from 1965 Russian ed.)

20. Desroches, J., Detournay, E., Lenoach, B., Papanastasiou, P., Pearson, J.R.A., Thiercelin, M., Cheng, A.H-D.: The crack tip region in hydraulic fracturing. Proc. R. Soc. Lond. Series A 447, 39-48 (1994) 